\title{
$m$-order integrals and generalized Itô's formula; the case of a fractional Brownian motion with any Hurst index
}

\author{
Mihai Gradinaru ${ }^{\mathrm{a}, *}$, Ivan Nourdin ${ }^{\mathrm{a}}$, Francesco Russo ${ }^{\mathrm{b}}$, Pierre Vallois ${ }^{\text {a }}$ \\ ${ }^{a}$ Université Henri Poincaré, institut de mathématiques Élie Cartan, B.P. 239, 54506 Vandouvre-lès-Nancy Cedex, France \\ ${ }^{\mathrm{b}}$ Université Paris 13, institut Galilée, mathématiques, 99, avenue J.B. Clément, 93430 Villetaneuse Cedex, France
}

Received 15 October 2003; received in revised form 10 April 2004; accepted 1 June 2004

Available online 18 September 2004

\begin{abstract}
Given an integer $m$, a probability measure $v$ on $[0,1]$, a process $X$ and a real function $g$, we define the $m$-order $v$-integral having as integrator $X$ and as integrand $g(X)$. In the case of the fractional Brownian motion $B^{H}$, for any locally bounded function $g$, the corresponding integral vanishes for all odd indices $m>\frac{1}{2 H}$ and any symmetric $v$. One consequence is an ItôStratonovich type expansion for the fractional Brownian motion with arbitrary Hurst index $H \in] 0,1[$. On the other hand we show that the classical Itô-Stratonovich formula holds if and only if $H>\frac{1}{6}$.
\end{abstract}

(c) 2004 Elsevier SAS. All rights reserved.

\section{Résumé}

Un entier $m$, une mesure de probabilité $v$ sur $[0,1]$, un processus $X$ et une fonction réelle $g$ étant donnés, on définit une $\nu$-intégrale d'ordre $m$ ayant $X$ comme intégrateur et $g(X)$ comme intégrand. Dans le cas du mouvement brownien fractionnaire $B^{H}$, on prouve, pour toute fonction localement bornée $g$, que l'intégrale correspondante s'annule pour tous les indices $m>$ $\frac{1}{2 H}$ et pour toutes les mesures symétriques $v$. Comme conséquence, on obtient une formule de type Itô-Stratonovich pour le mouvement brownien fractionnaire d'indice de Hurst quelconque dans ]0, 1[. D'autre part, on montre que la formule d'ItôStratonovich est valide si et seulement si $H>\frac{1}{6}$.

(c) 2004 Elsevier SAS. All rights reserved.

MSC: 60H05; 60G15; 60G18

Keywords: m-order integral; Itô's formula; Fractional Brownian motion

\footnotetext{
* Corresponding author.

E-mail address: Mihai.Gradinaru@iecn.u-nancy.fr (M. Gradinaru).
} 


\section{Introduction}

The present paper is devoted to $m$-order $v$-integrals and an Itô's formula for non-semimartingales. Classical Itô's formula and classical covariations are fundamental tools of stochastic calculus with respect to semimartingales. Calculus involving integrators $X$ which are not semimartingales has been developed essentially in three directions in the last twenty years:

- The case when $X$ is a Dirichlet process.

- The case when $X$ is a Gaussian process.

- The case when $X$ has paths with $p$-variation greater than 2 .

The implemented techniques for this purpose have been of different natures: the Dirichlet forms approach, the Malliavin (or white noise) calculus approach through the theory of Skorohod integral, the Lyons rough path approach and the discretization-regularization approach.

It is impossible to list here all the contributors in previous topics; nevertheless we try to sketch some related short history; a survey with a more complete literature could be found in [15].

1. A Dirichlet process may be seen as a natural generalization of a semimartingale: it is constituted by the sum of a local martingale and a zero quadratic variation (instead of a finite variation) process. Such a process is in particular a finite quadratic variation process. Calculus with respect to Dirichlet processes has been developed within two axes. One uses the Dirichlet forms approach, from which the term Dirichlet process was inspired: a fairly complete monography on the subject can be found in [13]. In this framework one can quote for instance $[18,17,26]$. The second approach uses the discretization of the integrals (see e.g. $[11,12,7,10]$ ). A counterpart of this approach is the regularization approach (see e.g. [22-24,8,14,27,29]). In particular those authors make use of the forward integral, which is a natural generalization of Itô integral, and the symmetric integral, which is a natural extension of Stratonovich integral. For those definitions, we refer to Section 2.

2. The Skorohod integral, and more generally the Malliavin calculus (see e.g. [20]), has been revealed to be a good tool for considering Gaussian integrators, and in particular fractional Brownian motion. For illustration we quote [6,1] and [21] for the case of $X$ being itself a Skorohod integral.

3. The rough path approach has been performed by T. Lyons [16], and continued by several authors; among them, [5] has adapted this technique to the study of SDEs driven by fractional Brownian motion.

The regularization approach has been recently continued by $[9,15]$ to analyze calculus with respect to integrands whose $n$-variation is greater than 2, developing the notion of $n$-covariation. In particular, [9] introduces the notion of 3-variation (or cubic variation) of a process, denoted by $[X, X, X]$.

We come back now to the main application of this paper, that is fractional Brownian motion. This process, which in general is not a semimartingale, has been studied intensively in stochastic analysis and it is considered in many applications, e.g. in hydrology, telecommunications, fluidodynamics, economics and finance.

Recall that a mean zero Gaussian process $X=B^{H}$ is a fractional Brownian motion with Hurst index $\left.H \in\right] 0,1[$ if its covariance function is given by

$$
K_{H}(s, t)=\frac{1}{2}\left(|s|^{2 H}+|t|^{2 H}-|s-t|^{2 H}\right), \quad(s, t) \in \mathbb{R}^{2} .
$$

An easy consequence of that property is that

$$
\mathrm{E}\left(B_{t}^{H}-B_{s}^{H}\right)^{2}=|t-s|^{2 H} .
$$

When $H=\frac{1}{2}, B^{H}$ is the classical Brownian motion. It is well-known that $B^{H}$ is a semimartingale if and only if $H=\frac{1}{2}$. On the other hand, if $H>\frac{1}{2}, B^{H}$ is a zero quadratic variation process, therefore (trivially) also a Dirichlet 
process. As we said, if $H \geqslant \frac{1}{2}, B^{H}$ is a finite quadratic variation process, therefore an Itô's formula involving symmetric integrals holds, and it can be deduced from [23,11]. If $f$ is of class $\mathrm{C}^{2}$, we have

$$
f\left(B_{t}^{H}\right)=f\left(B_{0}^{H}\right)+\int_{0}^{t} f^{\prime}\left(B_{s}^{H}\right) d^{\circ} B_{s}^{H} .
$$

If $H>\frac{1}{2},\left[B^{H}, B^{H}\right]$ vanishes and the symmetric integral $\int_{0}^{t} f^{\prime}\left(B_{s}^{H}\right) d^{\circ} B_{s}^{H}$ coincides with the forward integral $\int_{0}^{t} f^{\prime}\left(B_{s}^{H}\right) d^{-} B_{s}^{H}$.

Setting $f(x)=x^{2},(1.3)$ says that

$$
\left(B_{t}^{H}\right)^{2}=\left(B_{0}^{H}\right)^{2}+2 \int_{0}^{t} B_{s}^{H} d^{\circ} B_{s}^{H} .
$$

If $H<\frac{1}{2}$ the forward integral $\int_{0}^{t} B_{s}^{H} d^{-} B_{s}^{H}$ does not exist, but (1.4) is still valid. In fact, using the identity

$$
\left(B_{s+\varepsilon}^{H}\right)^{2}=\left(B_{s}^{H}\right)^{2}+2 \frac{B_{s+\varepsilon}^{H}+B_{s}^{H}}{2}\left(B_{s+\varepsilon}^{H}-B_{s}^{H}\right),
$$

integrating from zero to $t$ both members of the equality, dividing by $\varepsilon$ and using the definition of symmetric integral, we can immediately see that (1.4) holds for any $0<H<1$. The natural question which arises is the following: is (1.3) valid for any $0<H<1$ ? The answer is no. In reality, taking $f(x)=x^{3}$, similarly to (1.5), we can expand as follows

$$
\left(B_{s+\varepsilon}^{H}\right)^{3}=\left(B_{s}^{H}\right)^{3}+3 \frac{\left(B_{s+\varepsilon}^{H}\right)^{2}+\left(B_{s}^{H}\right)^{2}}{2}\left(B_{s+\varepsilon}^{H}-B_{s}^{H}\right)-\frac{\left(B_{s+\varepsilon}^{H}-B_{s}^{H}\right)^{3}}{2} .
$$

Proceeding as before, $\left(B_{t}^{H}\right)^{3}$ could be expanded as

$$
\left(B_{t}^{H}\right)^{3}=\left(B_{0}^{H}\right)^{3}+3 \int_{0}^{t}\left(B_{s}^{H}\right)^{2} d^{\circ} B_{s}^{H}-\frac{\left[B^{H}, B^{H}, B^{H}\right]_{t}}{2} ;
$$

moreover previous symmetric integral will exist if and only if $\left[B^{H}, B^{H}, B^{H}\right]$ exists.

In reality, that object exists if and only if $H>\frac{1}{6}$ : in that case the mentioned cubic variation even vanishes. This point comes out as a consequence of Theorem 4.12. when $m=3$. This shows that the Itô-Stratonovich formula (1.3) cannot be extended to the case $H \leqslant \frac{1}{6}$. On the other hand this observation asks the following important question: is (1.3) correct for all $H>\frac{1}{6}$ ?

In [15], one defined the forward (resp. backward, symmetric) integrals of order $m=3$, denoted by $\int_{0}^{t} Y_{S} d^{-(m)} X_{S}$ (resp. $\left.\int_{0}^{t} Y_{S} d^{+(m)} X_{s}, \int_{0}^{t} Y_{S} d^{\circ(m)} X_{S}\right)$. Given a locally bounded function $g$, there, it was proved that the forward integral of third order type $\int_{0}^{t} g\left(B_{s}^{H}\right) d^{(-3)} B_{s}^{H}$ exists for $H \geqslant \frac{1}{4}$. More precisely, if $H>\frac{1}{4}, \int_{0}^{t} g\left(B_{s}^{H}\right) d^{-(3)} B_{s}^{H}$ vanishes; if $H=\frac{1}{4}, \int_{0}^{t} g\left(B_{s}^{1 / 4}\right) d^{-(3)} B_{S}^{1 / 4}$ is non-zero and it is related to the local time of $B^{1 / 4}$; if, furthermore, $g$ is of class $\mathrm{C}^{1}$, we have

$$
\int_{0}^{t} g\left(B_{s}^{\frac{1}{4}}\right) d^{-(3)} B_{s}^{\frac{1}{4}}=-\frac{3}{2} \int_{0}^{t} g^{\prime}\left(B_{s}^{\frac{1}{4}}\right) d s .
$$

In particular, one deduced that the 3-order symmetric integral $\int_{0}^{t} g\left(B_{s}^{H}\right) d^{\circ(3)} B_{s}^{H}$ exists and vanishes for $H \geqslant \frac{1}{4}$. However, it is possible to see that for $H \leqslant \frac{1}{4}$, previous forward 3-order integral does not exist in general, see Theorem 4.11 . 
In this paper, we can show that the previous symmetric 3-order integral still exists (and vanishes) for $H>\frac{1}{6}$. This allows us to extend Itô's formula (1.3) to the case $H>\frac{1}{6}$ and to show that $H=\frac{1}{6}$ is a barrier for validity of formula (1.3). We also investigate the existence of symmetric $m$-order integrals for $m \geqslant 3$. We have to distinguish two cases, according to the evenness of $m$.

- if $2 n H>1$ then integral $\int_{0}^{t} g\left(B_{u}^{H}\right) d^{\circ(2 n)} B_{u}^{H}$ exists and vanishes;

- if $(2 n-1) H>\frac{1}{2}$ then integral $\int_{0}^{t} g\left(B_{u}^{H}\right) d^{\circ(2 n-1)} B_{u}^{H}$ exists and vanishes;

see Theorem 4.1 for a precise statement. We prove that we can not go further if $2 n H<1$ or $(2 n-1) H<\frac{1}{2}$, since the integrals above do not exist. We also investigate the cases $2 n H=1$ and $(2 n-1) H=\frac{1}{2}$.

Next natural question is the following. Is it possible to extend somehow (1.3) if $H \leqslant \frac{1}{6}$ ? For this purpose, we prove Theorem 3.6 which gives a general Itô's expansion of pathwise type and we establish the important Theorem 4.4.2 for the fractional Brownian motion with Hurst index $0<H<1$.

One relevant feature of this paper, is the definition of a new class of integrals. Let $X$ be a continuous process. Given a positive integer $m$ and a probability measure $v$ on $[0,1]$, we introduce the following $m$-order $v$-integral of $g(X)$ with respect to $X, g$ being a locally bounded Borel fonction:

$$
\int_{0}^{t} g\left(X_{u}\right) d^{v, m} X_{u}:=\lim _{\varepsilon \downarrow 0} \operatorname{prob} \frac{1}{\varepsilon} \int_{0}^{t} d u\left(X_{u+\varepsilon}-X_{u}\right)^{m} \int_{0}^{1} g\left(X_{u}+\alpha\left(X_{u+\varepsilon}-X_{u}\right)\right) v(d \alpha) .
$$

If $X$ is a continuous semimartingale and $v$ is a probability measure on $[0,1]$, these integrals were introduced in [28] for the case $m=1$.

A $m$-order forward (resp. backward and symmetric) integral of $g(X)$ with respect to $X$ can be expressed in the framework of $m$-order $v$-integral, with $v=\delta_{0}$, the Dirac measure at 0 (resp. $\left.\delta_{1}, \frac{\delta_{0}+\delta_{1}}{2}\right)$.

When $v$ is symmetric the corresponding integral is a natural extension of symmetric integrals of Stratonovich type. Proposition 3.3 characterizes that integral in terms of a sum of integrals involving $g^{(k)}(X)$ as integrand and $v=\delta_{1 / 2}$ and Theorem 3.6 establishes a general Itô's expansion. The probability measure $v$ may also be absolutely continuous, but it will be less interesting: if $v$ is Lebesgue measure, the integral becomes trivial.

Section 4 is devoted to applications with respect to fractional Brownian motion. There we distinguish two main levels of results.

- The Itô-Stratonovich formula (1.3) can be extended to $H>\frac{1}{6}$ (Theorem 4.41 and Remark 4.51 ) and cannot be improved.

- If $H \leqslant \frac{1}{6}$, it is still possible to expand $f\left(B_{t}^{H}\right)$ through a pathwise type Itô formula. It is the aim of Theorem 4.4.2 which considers an integral of a $v$-symmetric integral being in fact a renormalized Stratonovich integral.

We conclude this introduction insisting on the novelties introduced by this paper with respect to some recent contributions.

1. Concerning Itô formula for $B^{H}$ with respect to any $0<H<1$, there are contributions when the driving integral is an extend Skorohod integral, see for instance [2,4] and [3] which has emphasized a $\frac{1}{6}$ as a critical value in their framework.

2. At our knowledge, this is the first paper which treats an Itô formula with respect to a symmetric-Stratonovich integral, which is closer to the spirit of Riemann sums limits. We define for that purpose a class of high order integrals, which, from our point of view, have an interest by themselves.

3. We are able to treat an Itô formula with respect to somehow any symmetric integral, introducing a large class of symmetric integrals via regularization, i.e. $\int_{0}^{t} g\left(B_{u}^{H}\right) d^{v, 1} B_{u}^{H}$. Moreover we are able to treat all the pathologies 
related to such Itô formula. For instance, an Itô formula with respect to the classical symmetric integral only holds for any $H>\frac{1}{6}$.

4. When $H \leqslant \frac{1}{6}$, our procedure is inspired by numerical analysis and provides the exact renormalizations we need to allow convergence of our regularization scheme; a similar analysis could be adapted using a discretization approach.

5. Fractional Brownian motion is not the only process for which our Itô formula is valid; there are easy extensions to a more general class of processes. We believe however that fractional Brownian motion is a peculiar example for formulating necessary and sufficient conditions, through the Hurst parameter which guides the regularity of paths.

\section{Notations and recalls of preliminary results}

We start recalling some definitions and results established in some previous papers, see [22-24]. In the following $X$ (resp. $Y$ ) will be a continuous (resp. locally bounded) process. The space of continuous processes will be a metrizable Fréchet space $\mathcal{C}$, if it is endowed with the topology of the uniform convergence in probability on each compact interval (ucp). The space of random variables is also a metrizable Fréchet space, denoted by $\mathrm{L}^{0}(\Omega)$ and it is equipped with the topology of the convergence in probability.

The forward integral and the covariation are respectively defined by

$$
\int_{0}^{t} Y_{u} d^{-} X_{u}:=\lim _{\varepsilon \downarrow 0} \operatorname{ucp} \frac{1}{\varepsilon} \int_{0}^{t} Y_{u}\left(X_{u+\varepsilon}-X_{u}\right) d u
$$

and

$$
[X, Y]_{t}:=\lim _{\varepsilon \downarrow 0} \operatorname{ucp} \frac{1}{\varepsilon} \int_{0}^{t}\left(X_{u+\varepsilon}-X_{u}\right)\left(Y_{u+\varepsilon}-Y_{u}\right) d u .
$$

The symmetric (Stratonovich) integral is defined as

$$
\int_{0}^{t} Y_{u} d^{\circ} X_{u}:=\lim _{\varepsilon \downarrow 0} \operatorname{ucp} \frac{1}{\varepsilon} \int_{0}^{t} \frac{Y_{u+\varepsilon}+Y_{u}}{2}\left(X_{u+\varepsilon}-X_{u}\right) d u .
$$

The following fundamental equality is valid

$$
\int_{0}^{t} Y_{u} d^{\circ} X_{u}=\int_{0}^{t} Y_{u} d^{-} X_{u}+\frac{1}{2}[X, Y]_{t},
$$

provided that two quantities among three exist. However, as we will see in the next section, the left member may exist even if the covariation $[X, Y]$ does not exist.

If $X$ is such that $[X, X]$ exists, $X$ is called finite quadratic variation process. If $[X, X]=0$, then $X$ will be called zero quadratic variation process. In particular a Dirichlet process is a finite quadratic variation process. If $X$ is finite quadratic variation process and if $f \in \mathrm{C}^{2}(\mathbb{R})$, then the following Itô's formula holds:

$$
f\left(X_{t}\right)=f\left(X_{0}\right)+\int_{0}^{t} f^{\prime}\left(X_{u}\right) d^{-} X_{u}+\frac{1}{2}\left[f^{\prime}(X), X\right]_{t} .
$$


We recall that finite quadratic variation processes are stable through $\mathrm{C}^{1}$-transformations. In particular, if $f, g \in$ $\mathrm{C}^{1}$ and vector $(X, Y)$ is such that all mutual covariations $[X, X],[X, Y]$ and $[Y, Y]$ exist, then $[f(X), g(Y)]_{t}=$ $\int_{0}^{t} f^{\prime}\left(X_{S}\right) g^{\prime}\left(Y_{S}\right) d[X, Y]_{s}$. Hence, formulae (2.4) and (2.5) give:

$$
f\left(X_{t}\right)=f\left(X_{0}\right)+\int_{0}^{t} f^{\prime}\left(X_{u}\right) d^{\circ} X_{u} .
$$

\section{Remark 2.1.}

1. If $X$ is a continuous semimartingale and $Y$ is a suitable previsible process, then $\int_{0}^{\cdot} Y_{u} d^{-} X_{u}$ is classical Itô's integral.

2. If $X$ and $Y$ are (continuous) semimartingales then $\int_{0}^{\cdot} Y_{u} d^{\circ} X_{u}$ is the Fisk-Stratonovich integral and $[X, Y]$ is the ordinary square bracket.

3. If $X=B^{H}$, then its paths are a.s. Hölder continuous with parameter strictly less than $H$. Therefore it is easy to see that, if $H>\frac{1}{2}$, then $B^{H}$ is a zero quadratic variation process. When $H=\frac{1}{2}, B=B^{1 / 2}$ is the classical Brownian motion and so $\left[B^{1 / 2}, B^{1 / 2}\right]_{t}=t$. In particular Itô's formula (2.6) holds for $H \geqslant \frac{1}{2}$.

Since the quadratic variation is not defined for $B^{H}$ when $H<\frac{1}{2}$, we need to find a substitution tool. A concept of $\alpha$-variation was already introduced in [24]. Here it will be called strong $\alpha$-variation and it is the following increasing continuous process:

$$
[X]_{t}^{(\alpha)}:=\lim _{\varepsilon \downarrow 0} \operatorname{ucp} \int_{0}^{t} \frac{\left|X_{u+\varepsilon}-X_{u}\right|^{\alpha}}{\varepsilon} d u .
$$

A real attempt to adapt previous approach to integrators $X$ which are not of finite quadratic variation has been done in [9]. For a positive integer $n$, in [9] one defines the $n$-covariation $\left[X^{1}, \ldots, X^{n}\right]$ of a vector $\left(X^{1}, \ldots, X^{n}\right)$ of real continuous processes, in the following way:

$$
\left[X^{1}, \ldots, X^{n}\right]_{t}:=\lim _{\varepsilon \downarrow 0} \operatorname{ucp} \int_{0}^{t} \frac{\left(X_{u+\varepsilon}^{1}-X_{u}^{1}\right) \ldots\left(X_{u+\varepsilon}^{n}-X_{u}^{n}\right)}{\varepsilon} d u .
$$

Clearly, if $n=2$, the 2-covariation $\left[X^{1}, X^{2}\right]$ is the covariation previously defined. In particular, if all the processes $X^{i}$ are equal to $X$ than the definition gives:

$$
\underbrace{[X, \ldots, X]}_{n \text { times }}(t):=\lim _{\varepsilon \downarrow 0} \operatorname{ucp} \int_{0}^{t} \frac{\left(X_{u+\varepsilon}-X_{u}\right)^{n}}{\varepsilon} d u,
$$

which is called the $n$-variation of process $X$.

Remark 2.2. Clearly, for even integer $2 n$,

$$
[X]^{(2 n)}=\underbrace{[X, \ldots, X]}_{2 n \text { times }} .
$$

For this reason, in the sequel, if we formulate the assumption that the (2n)-variation of $X$ exists, that will mean that the strong $(2 n)$-variation of $X$ exists.

The following properties have been established in [9]. 


\section{Remark 2.3.}

1. If the strong $n$-variation of $X$ exists, then for all $m>n,[X]^{(m)}$ and $\underbrace{[X, \ldots, X]}_{m \text { times }}$ exist and vanish.

2. If $\underbrace{[X, \ldots, X]}_{n \text { times }}$ and $[X]^{(n)}$ exist then, for $g \in \mathrm{C}(\mathbb{R})$,

$$
\lim _{\varepsilon \downarrow 0} \operatorname{ucp} \int_{0}^{t} g\left(X_{u}\right) \frac{\left(X_{u+\varepsilon}-X_{u}\right)^{n}}{\varepsilon} d u=\int_{0}^{t} g\left(X_{u}\right) d[X, X, \ldots, X]_{u} .
$$

Furthermore, if $f_{1}, \ldots, f_{n} \in \mathrm{C}^{1}(\mathbb{R})$, then

$$
\underbrace{\left[f_{1}(X), \ldots, f_{n}(X)\right]}_{n \text { times }}(t)=\int_{0}^{t} f_{1}^{\prime}\left(X_{u}\right) \ldots f_{n}^{\prime}\left(X_{u}\right) d \underbrace{[X, \ldots, X]}_{n \text { times }}(u) .
$$

3. Let us come back to the process $X=B^{H}$. If $H \geqslant \frac{1}{3}$, its strong 3-variation $\left[B^{H}\right]^{(3)}$ exists and its 3-variation $\left[B^{H}, B^{H}, B^{H}\right]$ exists and vanishes. In [15], it is proved that

$$
\lim _{\varepsilon \downarrow 0} \operatorname{prob} \int_{0}^{t} \frac{\left(B_{u+\varepsilon}^{H}-B_{u}^{H}\right)^{3}}{\varepsilon} d u
$$

exists and vanishes, even for $H>\frac{1}{6}$.

Remark 2.4. In [24, Proposition 3.14, p. 22], it is proved that the strong $\frac{1}{H}$-variation of $B^{H}$ exists and equals $\mu_{\frac{1}{H}} t$, where $\mu_{a}=\mathrm{E}\left[|G|^{a}\right]$, with $G$ a standard Gaussian random variable. For instance,

$$
\left[B^{H}\right]_{t}^{(2 n)}= \begin{cases}\mu_{2 n} t, & \text { if } H=\frac{1}{2 n}, \\ 0, & \text { if } H>\frac{1}{2 n} .\end{cases}
$$

Proposition 2.5. Let $n$ be a positive integer. If $B^{H}$ is a fractional Brownian motion with Hurst index $\left.H \in\right] 0,1[$ then

$$
[\underbrace{\left[g\left(B^{H}\right), B^{H}, \ldots, B^{H}\right.}_{2 n}]_{t}=\mu_{2 n} \begin{cases}\int_{0}^{t} g^{\prime}\left(B_{s}^{H}\right) d s, & \text { if } H=\frac{1}{2 n} \\ 0, & \text { if } H>\frac{1}{2 n} .\end{cases}
$$

Proof. It is a consequence of Remark 2.3 2. and Remark 2.4.

Remark 2.6. From now on we relax the definitions of $n$-covariation and $n$-variation in the sense that the limits (2.8) and (2.9) are assumed to hold in probability and the limiting $\mathrm{L}^{0}(\Omega)$-valued functions have continuous versions. Nevertheless, for even integers $2 n$, the existence of the $(2 n)$-variation of the process $X$ (in this weaker sense) implies the strong existence (that is as an ucp limit). This follows by the Dini type result constituted by Lemma 3.1 in [24]: this says that if a sequence of increasing continuous processes converges in probability at each time toward a continous process, then the convergence actually holds uniformly in probability on each compact interval of time (ucp). In the sequel this remark will be used without further comment.

A natural extension of (2.1) and (2.3) is the following. 
Definition 2.7. Let $X$ (resp. $Y$ ) be a continuous (resp. locally bounded) processes. Let $m \geqslant 1$.

We denote

$$
\int_{0}^{t} Y_{u} d^{\circ(m)} X_{u}=\lim _{\varepsilon \downarrow 0} \operatorname{prob} \frac{1}{\varepsilon} \int_{0}^{t} \frac{Y_{u}+Y_{u+\varepsilon}}{2}\left(X_{u+\varepsilon}-X_{u}\right)^{m} d u ;
$$

this quantity is called (definite) symmetric integral of $m$-order type of $Y$ with respect to $X$.

Similarly we can define the $m$-order integral of forward (resp. backward) type.

We set

$$
\int_{0}^{t} Y_{u} d^{-(m)} X_{u}=\lim _{\varepsilon \downarrow 0} \operatorname{prob} \frac{1}{\varepsilon} \int_{0}^{t} Y_{u}\left(X_{u+\varepsilon}-X_{u}\right)^{m} d u ;
$$

this quantity is called (definite) forward integral of $m$-order type of $Y$ with respect to $X$. The backward $m$-order (definite) integral will be defined as follows

$$
\int_{0}^{t} Y_{u} d^{+(m)} X_{u}=\lim _{\varepsilon \downarrow 0} \operatorname{prob} \frac{1}{\varepsilon} \int_{0}^{t} Y_{u+\varepsilon}\left(X_{u+\varepsilon}-X_{u}\right)^{m} d u .
$$

Remark 2.8. (a) We have

$$
\int_{0}^{t} Y_{u} d^{\circ(1)} X_{u}=\int_{0}^{t} Y_{u} d^{\circ} X_{u}, \quad \int_{0}^{t} Y_{u} d^{-(1)} X_{u}=\int_{0}^{t} Y_{u} d^{-} X_{u} .
$$

(b) If $X$ is a finite quadratic variation process, then $\int_{0}^{t} Y_{u} d^{\circ(2)} X_{u}=\int_{0}^{t} Y_{u} d[X]_{u}$.

(c) If $X=B^{H}, H \geqslant \frac{1}{4}, g \in \mathrm{C}(\mathbb{R})$, then $\int_{0}^{t} g\left(B_{u}^{H}\right) d^{\circ(3)} B_{u}^{H}=0$.

(a) and (b) are straightforward, see [22]. The proof of (c) was performed in [15], showing separately the existence of the 3-forward integral, which in some cases is nonzero, see also (1.7).

Remark 2.9. Let $n, m \geqslant 1$, be two integers. Provided that two quantities among three exist, the third exists and the following equalities hold:

$$
\begin{aligned}
& \text { (a) }[\underbrace{Y, X, \ldots, X}_{2 n}]_{t}=\int_{0}^{t} Y_{u} d^{+(2 n-1)} X_{u}-\int_{0}^{t} Y_{u} d^{-(2 n-1)} X_{u} \text {, } \\
& \text { (b) } \int_{0}^{t} Y_{u} d^{\circ(m)} X_{u}=\frac{1}{2}\left[\int_{0}^{t} Y_{u} d^{+(m)} X_{u}+\int_{0}^{t} Y_{u} d^{-(m)} X_{u}\right] \text {. }
\end{aligned}
$$

In general forward and backward integrals $\int_{0}^{t} Y_{u} d^{ \pm(m)} X_{u}$ do not exist, while the symmetric integral $\int_{0}^{t} Y_{u} d^{\circ(m)} X_{u}$ may exist.

\section{3. $m$-order $v$-integrals and Itô's formula}

We start here defining the concept of $m$-order $v$-integrals; when the integrator is a semimartingale and in the case $m=1$, this concept has been defined by [28, p. 521]. As previously, $X$ will be a continuous process. Henceforth, $v$ will denote a probability measure on $[0,1]$. We shall denote 


$$
m_{k}:=\int_{0}^{1} \alpha^{k} v(d \alpha)
$$

the $k$ th moment of $v$.

Definition 3.1. Let $m$ be a positive integer. For a locally bounded function $g: \mathbb{R} \rightarrow \mathbb{R}$, the $m$-order $v$-integral of $g(X)$ with respect to $X$ is given by

$$
\int_{0}^{t} g\left(X_{u}\right) d^{v, m} X_{u}=\lim _{\varepsilon \downarrow 0} \operatorname{prob} \frac{1}{\varepsilon} \int_{0}^{t} d u\left(X_{u+\varepsilon}-X_{u}\right)^{m} \int_{0}^{1} g\left(X_{u}+\alpha\left(X_{u+\varepsilon}-X_{u}\right)\right) v(d \alpha) .
$$

Remark 3.2. This integral with respect to $X$ is only defined for integrands of the type $g(X)$. Nevertheless, in some cases, see for instance (b), (c), (d) below, we can take an arbitrary integrand $Y$.

For example, we have the following.

(a) If $g \equiv 1$ then, for any probability measure $v, \int_{0}^{t} d^{v, m} X_{u}$ is the $m$-variation of $X$, see (2.9).

(b) If $v=\delta_{0}$ and $m \in \mathbb{N}^{*}$, then $\int_{0}^{t} g\left(X_{u}\right) d^{\nu, m} X_{u}$ is the forward integral of $m$-order type, see Definition 2.7.

(c) If $v=\delta_{1}$ and $m \in \mathbb{N}^{*}$, then $\int_{0}^{t} g\left(X_{u}\right) d^{v, m} X_{u}$ is the backward integral of $m$-order type, see Definition 2.7.

(d) If $v=\frac{\delta_{0}+\delta_{1}}{2}$ and $m \in \mathbb{N}^{*}$, then $\int_{0}^{t} g\left(X_{u}\right) d^{v, m} X_{u}$ is the symmetric integral of $m$-order type, see Definition 2.7.

In the following, we continue to use notations

$$
\int_{0}^{t} g\left(X_{u}\right) d^{-(m)} X_{u} \quad\left(\text { resp. } \int_{0}^{t} g\left(X_{u}\right) d^{+(m)} X_{u}, \int_{0}^{t} g\left(X_{u}\right) d^{\circ(m)} X_{u}\right)
$$

instead of

$$
\int_{0}^{t} g\left(X_{u}\right) d^{\delta_{0}, m} X_{u} \quad\left(\operatorname{resp} . \int_{0}^{t} g\left(X_{u}\right) d^{\delta_{1}, m} X_{u}, \int_{0}^{t} g\left(X_{u}\right) d^{\frac{\delta_{0}+\delta_{1}}{2}, m} X_{u}\right) .
$$

The probability measure $v$ will be called symmetric if $v$ is invariant with respect to the map $t \mapsto 1-t$. For example, the probability measures $\delta_{1 / 2}, \frac{\delta_{0}+\delta_{1}}{2}$ and Lebesgue measure on $[0,1]$ are symmetric.

The symmetric probability measure $\delta_{1 / 2}$ plays a central role, as we can see by the following.

Proposition 3.3. Let $(k, m, n) \in \mathbb{N} \times \mathbb{N}^{*} \times \mathbb{N}^{*}$ be such that $k+m \geqslant 2 n$. Assume that $X$ has a ( $2 n$ )-variation $[X, X, \ldots, X]=[X]^{(2 n)}$ and $g \in C^{k}(\mathbb{R})$. If $v$ is a symmetric probability measure then, provided that all the integrals excepted one exist, the remaining one exists and we have

$$
\int_{0}^{t} g\left(X_{u}\right) d^{v, m} X_{u}=\sum_{i=0}^{\left[\frac{k-1}{2}\right]} \frac{m_{2 i}^{0}}{(2 i) !} \int_{0}^{t} g^{(2 i)}\left(X_{u}\right) d^{\delta_{1 / 2}, m+2 i} X_{u}+R_{t},
$$

with $m_{j}^{0}=\int_{0}^{1}\left(\frac{1}{2}-\alpha\right)^{j} v(d \alpha)$ and

$$
R_{t}= \begin{cases}0, & \text { if } k+m>2 n, \\ \frac{(-1)^{k} m_{0}^{k}}{k !} \int_{0}^{t} g^{(k)}\left(X_{u}\right) d[X, X, \ldots, X]_{u}, & \text { if } k+m=2 n .\end{cases}
$$




\section{Remark 3.4.}

1. If $k=0$ the sum in (3.2) is taken to be 0 . In this case

$$
\int_{0}^{t} g\left(X_{u}\right) d^{v, m} X_{u}= \begin{cases}0, & \text { if } m>2 n \\ \int_{0}^{t} g\left(X_{u}\right) d[X, X, \ldots, X]_{u}, & \text { if } m=2 n\end{cases}
$$

2. Note that $m_{j}^{0}$ equals zero for odd integers $j$ thanks to symmetry of $v$.

Proof of Proposition 3.3. (a) First, we prove (3.2) for the case when $k=0, m \geqslant 2 n+1$ and $g$ is bounded. Precisely, we prove that integrals $\int_{0}^{t} g\left(X_{u}\right) d^{v, m} X_{u}$ exist and vanish. We have almost surely:

$$
\left|\frac{1}{\varepsilon} \int_{0}^{t} d u\left(X_{u+\varepsilon}-X_{u}\right)^{m} \int_{0}^{1} g\left(X_{u}+\alpha\left(X_{u+\varepsilon}-X_{u}\right)\right) v(d \alpha)\right| \leqslant \frac{\text { cst. }}{\varepsilon} \int_{0}^{t}\left|X_{u+\varepsilon}-X_{u}\right|^{m} d u \rightarrow 0,
$$

as $\varepsilon \downarrow 0$, by Remark 2.3 part 1 . The convergence in probability follows.

(b) Second, we prove that integrals $\int_{0}^{t} g\left(X_{u}\right) d^{\nu, m} X_{u}$ exist and vanish when $m \geqslant 2 n+1$ but $g$ is only locally bounded. In this case, we perform the following localization argument, which will be used several times. Let $\beta>0$; we will show that

$$
\lim _{\varepsilon \downarrow 0} \mathrm{P}\left(\left|\frac{1}{\varepsilon} \int_{0}^{t} d u\left(X_{u+\varepsilon}-X_{u}\right)^{m} \int_{0}^{1} g\left(X_{u}+\alpha\left(X_{u+\varepsilon}-X_{u}\right)\right) v(d \alpha)\right| \geqslant \beta\right)=0 .
$$

Let $M>0, \Omega_{M}=\left\{\omega:\left|X_{u}(\omega)\right| \leqslant M ; \forall u \in[0, t+1]\right\}$. On $\Omega_{M}$, we have

$$
\begin{aligned}
& \frac{1}{\varepsilon} \int_{0}^{t} d u\left(X_{u+\varepsilon}-X_{u}\right)^{m} \int_{0}^{1} g\left(X_{u}+\alpha\left(X_{u+\varepsilon}-X_{u}\right)\right) v(d \alpha) \\
& \quad=\frac{1}{\varepsilon} \int_{0}^{t} d u\left(X_{u+\varepsilon}-X_{u}\right)^{m} \int_{0}^{1} g_{M}\left(X_{u}+\alpha\left(X_{u+\varepsilon}-X_{u}\right)\right) v(d \alpha)
\end{aligned}
$$

where $g_{M}=g 1_{[-M, M]}$.

We can write

$$
\begin{aligned}
& \mathrm{P}\left(\left|\frac{1}{\varepsilon} \int_{0}^{t} d u\left(X_{u+\varepsilon}-X_{u}\right)^{m} \int_{0}^{1} g\left(X_{u}+\alpha\left(X_{u+\varepsilon}-X_{u}\right)\right) v(d \alpha)\right| \geqslant \beta\right) \\
& \quad \leqslant \mathrm{P}\left(\left|\frac{1}{\varepsilon} \int_{0}^{t} d u\left(X_{u+\varepsilon}-X_{u}\right)^{m} \int_{0}^{1} g_{M}\left(X_{u}+\alpha\left(X_{u+\varepsilon}-X_{u}\right)\right) v(d \alpha)\right| \geqslant \beta\right)+\mathrm{P}\left(\Omega_{M}^{c}\right) .
\end{aligned}
$$

Take $\delta>0$; we choose $M$ large enough, so that $P\left(\Omega_{M}^{c}\right)<\frac{\delta}{2}$. By convergence in probability, for the bounded function $g_{M}$, there exists $\eta>0$ such that for each $\varepsilon<\eta$ the first term in previous inequality is less than $\frac{\delta}{2}$. We obtain the existence and the cancellation of $\int_{0}^{t} g\left(X_{u}\right) d^{v, m} X_{u}$. 
(c) For the general case, using a Taylor expansion, we can write:

$$
\begin{aligned}
g\left(X_{u}+\alpha\left(X_{u+\varepsilon}-X_{u}\right)\right)= & g\left(\frac{X_{u+\varepsilon}+X_{u}}{2}-\left(\frac{1}{2}-\alpha\right)\left(X_{u+\varepsilon}-X_{u}\right)\right) \\
= & \sum_{i=0}^{k-1} \frac{(-1)^{i}\left(\frac{1}{2}-\alpha\right)^{i}}{i !} g^{(i)}\left(\frac{X_{u+\varepsilon}+X_{u}}{2}\right)\left(X_{u+\varepsilon}-X_{u}\right)^{i} \\
& +(-1)^{k} \frac{\left(\frac{1}{2}-\alpha\right)^{k}}{k !} g^{(k)}\left(\theta_{\alpha}\right)\left(X_{u+\varepsilon}-X_{u}\right)^{k}
\end{aligned}
$$

with $\theta_{\alpha}$ between $X_{u}$ and $X_{u+\varepsilon}$. Since $m_{2 i+1}^{0}=0$ for integers $i$, we deduce,

$$
\begin{aligned}
& \frac{1}{\varepsilon} \int_{0}^{t}\left(\int_{0}^{1} g\left(X_{u}+\alpha\left(X_{u+\varepsilon}-X_{u}\right)\right) v(d \alpha)\right)\left(X_{u+\varepsilon}-X_{u}\right)^{m} d u \\
& =\sum_{i=0}^{\left[\frac{k-1}{2}\right]} \frac{m_{2 i}^{0}}{(2 i) !} \frac{1}{\varepsilon} \int_{0}^{t} g^{(2 i)}\left(\frac{X_{u+\varepsilon}+X_{u}}{2}\right)\left(X_{u+\varepsilon}-X_{u}\right)^{m+2 i} d u \\
& \quad+(-1)^{k} \frac{1}{k !} \int_{0}^{t}\left(\int_{0}^{1} g^{(k)}\left(\theta_{\alpha}\right)\left(\frac{1}{2}-\alpha\right)^{k} v(d \alpha)\right) \frac{\left(X_{u+\varepsilon}-X_{u}\right)^{k+m}}{\varepsilon} d u .
\end{aligned}
$$

We can assume that $g^{(k)}$ is bounded, by localization argument, as previously. Therefore last term on the right-hand side tends to $R$ ucp using Remark 2.3. The proof of the proposition is now established.

We can state now a straightforward (even though not very useful) Itô's formula, with very few assumptions.

Proposition 3.5. Assume that $v$ is the Lebesgue measure on $[0,1]$. If $f \in \mathrm{C}^{1}(\mathbb{R})$ and if $X$ is any continuous process, then the integral $\int_{0}^{t} f^{\prime}\left(X_{u}\right) d^{v, 1} X_{u}$ exists and we have:

$$
f\left(X_{t}\right)=f\left(X_{0}\right)+\int_{0}^{t} f^{\prime}\left(X_{u}\right) d^{v, 1} X_{u}
$$

Proof. Since $f$ belongs to $C^{1}(\mathbb{R})$, by classical Taylor formula:

$$
f\left(X_{u+\varepsilon}\right)=f\left(X_{u}\right)+\left(X_{u+\varepsilon}-X_{u}\right) \int_{0}^{1} f^{\prime}\left(X_{u}+\alpha\left(X_{u+\varepsilon}-X_{u}\right)\right) d \alpha .
$$

Integrating in $u$ on $[0, t]$ and dividing by $\varepsilon$, we obtain:

$$
\frac{1}{\varepsilon} \int_{t}^{t+\varepsilon} f\left(X_{u}\right) d u-\frac{1}{\varepsilon} \int_{0}^{\varepsilon} f\left(X_{u}\right) d u=\frac{1}{\varepsilon} \int_{0}^{t} d u\left(X_{u+\varepsilon}-X_{u}\right) \int_{0}^{1} f^{\prime}\left(X_{u}+\alpha\left(X_{u+\varepsilon}-X_{u}\right)\right) d \alpha .
$$

The left-hand side converges, as $\varepsilon \downarrow 0$, to $f\left(X_{t}\right)-f\left(X_{0}\right)$. Therefore, the right-hand side is also forced to have a limit in probability and equals $\int_{0}^{t} f^{\prime}\left(X_{u}\right) d^{v, 1} X_{u}$.

We are now able to state the main result of this section. 
Theorem 3.6 (Itô's formula). Let $n$ and $\ell$ two positive integers. Assume that $v$ is a symmetric probability measure on $[0,1]$ such that

$$
m_{2 j}:=\int_{0}^{1} \alpha^{2 j} v(d \alpha)=\frac{1}{2 j+1}, \quad \text { for } j=1, \ldots, \ell-1 .
$$

If $f \in \mathrm{C}^{2 n}(\mathbb{R})$ and if $X$ is a continuous process having a (2n)-variation, provided that all the integrals excepted one exist, the remaining exists and the following Itô formula holds:

$$
f\left(X_{t}\right)=f\left(X_{0}\right)+\int_{0}^{t} f^{\prime}\left(X_{u}\right) d^{v, 1} X_{u}+\sum_{j=\ell}^{n-1} k_{\ell, j}^{v} \int_{0}^{t} f^{(2 j+1)}\left(X_{u}\right) d^{\delta_{1 / 2}, 2 j+1} X_{u},
$$

where the sum is taken to be 0 for $\ell>n-1$. Here $k_{\ell, j}^{v}$ are suitable constants.

Remark 3.7. A significant application comes out when $v=\frac{\delta_{0}+\delta_{1}}{2}$. We obtain in that case

$$
f\left(X_{t}\right)=f\left(X_{0}\right)+\int_{0}^{t} f^{\prime}\left(X_{u}\right) d^{\circ} X_{u}+\sum_{j=1}^{n-1} k_{1, j}^{\nu} \int_{0}^{t} f^{(2 j+1)}\left(X_{u}\right) d^{\delta_{1 / 2}, 2 j+1} X_{u} .
$$

Proof of Theorem 3.6. Let us remark that (3.5) implies

$$
m_{j}=\frac{1}{j+1}, \quad j=1, \ldots, 2 \ell-1 .
$$

Indeed, we have

$$
m_{2 j+1}=\int_{0}^{1} \alpha^{2 j+1} v(d \alpha)=\int_{0}^{1}(1-\alpha)^{2 j+1} v(d \alpha)=\sum_{k=0}^{2 j+1}(-1)^{k} C_{2 j+1}^{k} m_{k},
$$

and, by induction,

$$
2 m_{2 j+1}=\sum_{k=0}^{2 j}(-1)^{k} C_{2 j+1}^{k} \frac{1}{k+1}=\frac{1}{j+1} .
$$

It suffices to prove that, for any $a, b \in \mathbb{R}$,

$$
\begin{aligned}
f(b)= & f(a)+(b-a) \int_{0}^{1} f^{\prime}(a+\alpha(b-a)) v(d \alpha) \\
& +\sum_{j=\ell}^{n-1} k_{\ell, j}^{\nu} f^{(2 j+1)}\left(\frac{a+b}{2}\right)(b-a)^{2 j+1}+(b-a)^{2 n} C(a, b),
\end{aligned}
$$

where $C \in \mathrm{C}\left(\mathbb{R}^{2}\right)$ verifies $C(a, a)=0$. Indeed, setting $a=X_{u}$ and $b=X_{u+\varepsilon}$, integrating in $u$ on $[0, t]$ and dividing by $\varepsilon$ we get:

$$
\frac{1}{\varepsilon} \int_{0}^{t}\left(f\left(X_{u+\varepsilon}\right)-f\left(X_{u}\right)\right) d u=\frac{1}{\varepsilon} \int_{0}^{t}\left(X_{u+\varepsilon}-X_{u}\right)\left(\int_{0}^{1} f^{\prime}\left(X_{u}+\alpha\left(X_{u+\varepsilon}-X_{u}\right)\right) v(d \alpha)\right) d u
$$




$$
\begin{aligned}
& +\sum_{j=\ell}^{n-1} k_{\ell, j}^{v} \frac{1}{\varepsilon} \int_{0}^{t} f^{(2 j+1)}\left(\frac{X_{u}+X_{u+\varepsilon}}{2}\right)\left(X_{u+\varepsilon}-X_{u}\right)^{2 j+1} d u \\
& +\frac{1}{\varepsilon} \int_{0}^{t} C\left(X_{u}, X_{u+\varepsilon}\right)\left(X_{u+\varepsilon}-X_{u}\right)^{2 n} d u .
\end{aligned}
$$

By a simple change of variable we can transform the left-hand side as

$$
\frac{1}{\varepsilon} \int_{t}^{t+\varepsilon} f\left(X_{u}\right) d u-\frac{1}{\varepsilon} \int_{0}^{\varepsilon} f\left(X_{u}\right) d u
$$

which tends as $\varepsilon \downarrow 0$, toward $f\left(X_{t}\right)-f\left(X_{0}\right)$. By the existence of the $(2 n)$-variation for $X$, since $\sup _{u \in[0, t]} C\left(X_{u}\right.$, $X_{u+\varepsilon}$ ) tends to zero, the last term on the right-hand side of the previous equality tends to zero, too. The convergence of all the terms excepted one on the right-hand side is insured by the hypothesis. Therefore, the term which remains on the right-hand side is also forced to have a limit in probability and we obtain (3.6). Hence, we need to prove formula (3.9). Thanks to Taylor expansions, we can write,

$$
\begin{aligned}
& f(b)-f(a)-(b-a) \int_{0}^{1} f^{\prime}(a+\alpha(b-a)) v(d \alpha) \\
& \quad=\sum_{i=1}^{2 n-1} f^{(i)}\left(\frac{a+b}{2}\right)(b-a)^{i}\left[\frac{1+(-1)^{i+1}}{i ! 2^{i}}-\frac{1}{(i-1) !} \int_{0}^{1}\left(\alpha-\frac{1}{2}\right)^{i-1} v(d \alpha)\right]+\mathrm{O}(b-a)^{2 n} .
\end{aligned}
$$

Moreover, since $v$ is symmetric, each integral $\int_{0}^{1}\left(\alpha-\frac{1}{2}\right)^{2 k+1} v(d \alpha)$ vanishes for $k=0, \ldots, n-1$. On the other hand, by using (3.8), easy computations allow to obtain that

$$
\frac{1}{(2 j) !} \int_{0}^{1}\left(\alpha-\frac{1}{2}\right)^{2 j} v(d \alpha)=\frac{1}{2^{2 j}(2 j+1) !}, \quad j=0, \ldots, \ell-1 .
$$

Finally, formula (3.9) follows and the proof of the theorem is concluded.

\section{The case of the fractional Brownian motion}

In this section, we investigate the existence of $\int_{0}^{t} g\left(X_{u}\right) d^{v, m} X_{u}$ when $X=B^{H}$ is a fractional Brownian motion with Hurst index $H \in] 0,1\left[\mu_{2 n}\right.$ will stand again for the $2 n$-moment of a standard Gaussian random variable.

Theorem 4.1. Let $m \geqslant 2$ be an integer and $v$ a probability measure on $[0,1]$.

1. Assume that $m=2 n$ and $g$ is a locally bounded function. Then

(a) if $2 n H \geqslant 1$ then $\int_{0}^{t} g\left(B_{u}^{H}\right) d^{v, 2 n} B_{u}^{H}$ exists and

$$
\int_{0}^{t} g\left(B_{u}^{H}\right) d^{v, 2 n} B_{u}^{H}=\int_{0}^{t} g\left(B_{u}^{H}\right) d\left[B^{H}\right]_{u}^{(2 n)}=\mu_{2 n} \begin{cases}\int_{0}^{t} g\left(B_{u}^{H}\right) d u, & \text { if } 2 n H=1, \\ 0, & \text { if } 2 n H>1 ;\end{cases}
$$

(b) if $2 n H<1$ then $\int_{0}^{t} g\left(B_{u}^{H}\right) d^{\nu, 2 n} B_{u}^{H}$ does not exist in general. 
2. Assume that $m=2 n+1$ and $g$ is locally bounded. Then

(a) if $(2 n+1) H>\frac{1}{2}$ then $\int_{0}^{t} g\left(B_{u}^{H}\right) d^{\delta_{1 / 2}, 2 n+1} B_{u}^{H}$ exists and vanishes;

(b) if $(2 n+1) H=\frac{1}{2}$ then $\frac{1}{\varepsilon} \int_{0}^{t}\left(B_{u+\varepsilon}^{H}-B_{u}^{H}\right)^{2 n+1} d u$ converges in law to a centered Gaussian random variable, as $\varepsilon \downarrow 0$;

(c) if $(2 n+1) H<\frac{1}{2}$ then $\int_{0}^{t} g\left(B_{u}^{H}\right) d^{\delta_{1 / 2}, 2 n+1} B_{u}^{H}$ does not exist in general.

3. Assume that $m=2 n+1, g$ belongs to $\mathrm{C}^{2 n+1}(\mathbb{R})$ and $v$ is symmetric. Then

(a) if $(2 n+1) H>\frac{1}{2}$ then $\int_{0}^{t} g\left(B_{u}^{H}\right) d^{v, 2 n+1} B_{u}^{H}$ exists and vanishes;

(b) if $(2 n+1) H<\frac{1}{2}$ then $\int_{0}^{t} g\left(B_{u}^{H}\right) d^{v, 2 n+1} B_{u}^{H}$ does not exist in general.

The proof of Theorem 4.1 is technical and it is postponed to the last section.

Corollary 4.2. Let $n$ be a positive integer, $g$ a continuous function and $t \geqslant 0$. Then, if $(2 n+1) H>\frac{1}{2}$, for all integers $\ell \geqslant n$, integrals $\int_{0}^{t} g\left(B_{u}^{H}\right) d^{\delta_{1 / 2}, 2 \ell+1} B_{u}^{H}$ exist and vanish.

For instance,

- if $H>\frac{1}{6}$, integrals $\int_{0}^{t} g\left(B_{u}^{H}\right) d^{\delta_{1 / 2}, \ell} B_{u}^{H}$ exists and vanishes for any odd integer $\ell \geqslant 3$;

- if $\frac{1}{10}<H \leqslant \frac{1}{6}$, integral $\int_{0}^{t} g\left(B_{u}^{H}\right) d^{\delta_{1 / 2}, 3} B_{u}^{H}$ does not exist in general, while integral $\int_{0}^{t} g\left(B_{u}^{H}\right) d^{\delta_{1 / 2}, \ell} B_{u}^{H}$ exists and vanishes for any odd integer $\ell \geqslant 5$.

Theorem 4.1 entails some results concerning non-symmetric integrals, see also (1.7).

Corollary 4.3. Let $n \in \mathbb{N}^{*}$ and $g \in \mathrm{C}^{2 n-1}(\mathbb{R})$. Assume that $B^{H}$ has a $(2 n)$-variation (i.e. $\left.2 n H \geqslant 1\right)$. Then the following $(2 n-1)$-order forward and backward integrals exist:

$$
\int_{0}^{t} g\left(B_{u}^{H}\right) d^{-(2 n-1)} B_{u}^{H}=-\int_{0}^{t} g\left(B_{u}^{H}\right) d^{+(2 n-1)} B_{u}^{H}= \begin{cases}0, & \text { if } 2 n H>1, \\ -\frac{\mu_{2 n}}{2} \int_{0}^{t} g^{\prime}\left(B_{u}^{H}\right) d u, & \text { if } 2 n H=1,\end{cases}
$$

where $\mu_{2 n}$ denotes the $2 n$-moment of a standard Gaussian random variable. In particular, $(2 n-1)$-order forward and backward integrals are not always equal to zero.

Proof. We choose $v=\frac{\delta_{0}+\delta_{1}}{2}$ in Theorem 4.1. Since $g \in \mathrm{C}^{2 n-1}(\mathbb{R})$ (see point 3.) and $(2 n-1) H>\frac{1}{2}$, we deduce $\int_{0}^{t} g\left(B_{u}^{H}\right) d^{\circ(2 n-1)} B_{u}^{H}=0 .(4.2)$ is now a consequence of Remark 2.9(a), (b) and Proposition 2.5.

We return now to Itô's formula for fractional Brownian motion. Theorem 3.6 says that, in the expansion of $f\left(B_{t}^{H}\right)$, the sum of stochastic integrals exists but each integral may be meaningless individually. What are the consequences in the applications to fractional Brownian motion? If we insist on working with symmetric Stratonovich integral, see (2.3), we are obliged to suppose $H>\frac{1}{6}$. However if $H \leqslant \frac{1}{6}$, an Itô's formula is still valid provided we proceed through a different regularization of the symmetric integral which involves particular symmetric probability measures.

\section{Theorem 4.4.}

1. If $H>\frac{1}{6}$ and $f \in \mathrm{C}^{6}(\mathbb{R})$, then the integral $\int_{0}^{t} f^{\prime}\left(B_{u}^{H}\right) d^{\nu, 1} B_{u}^{H}$ exists for any symmetric probability measure $v$ and we have

$$
f\left(B_{t}^{H}\right)=f(0)+\int_{0}^{t} f^{\prime}\left(B_{u}^{H}\right) d^{\nu, 1} B_{u}^{H} .
$$


2. Let $r \geqslant 2$ be an integer. If $(2 r+1) H>\frac{1}{2}$ and $f \in \mathrm{C}^{4 r+2}(\mathbb{R})$ then integral $\int_{0}^{t} f^{\prime}\left(B_{u}^{H}\right) d^{v, 1} B_{u}^{H}$ exists for any symmetric probability measure $v$ verifying

$$
m_{2 j}:=\int_{0}^{1} \alpha^{2 j} v(d \alpha)=\frac{1}{2 j+1}, \quad \text { for } j=1, \ldots, r-1 .
$$

Moreover, we have:

$$
f\left(B_{t}^{H}\right)=f\left(B_{0}^{H}\right)+\int_{0}^{t} f^{\prime}\left(B_{u}^{H}\right) d^{v, 1} B_{u}^{H} .
$$

Proof. If $H>\frac{1}{6}$, integrals $\int_{0}^{t} f^{(3)}\left(B_{u}^{H}\right) d^{\delta_{1 / 2}, 3} B_{u}^{H}$ and $\int_{0}^{t} f^{(5)}\left(B_{u}^{H}\right) d^{\delta_{1 / 2}, 5} B_{u}^{H}$ exist and vanish through Corollary 4.2. Theorem 3.6 applied to $n=3, \ell=1$ gives (4.3).

Again by Corollary 4.2, if $H>\frac{1}{4 r+2}$, integrals $\int_{0}^{t} f^{(2 \ell+1)}\left(B_{u}^{H}\right) d^{\delta_{1 / 2}, 2 \ell+1} B_{u}^{H}$ exist and vanish for $\ell \geqslant r$. Theorem 3.6 applied to $n=2 r+1, \ell=r$ and $v$ given by (4.6) entails (4.5).

\section{Remark 4.5.}

1. The symmetric probability measure $\frac{\delta_{0}+\delta_{1}}{2}$ satisfies $m_{2 j}=\frac{1}{2}$ for any integer $j \geqslant 1$. Consequently, the second part of previous theorem does not apply. However, by the first part, we have, for $H>\frac{1}{6}$ and $f \in \mathrm{C}^{6}(\mathbb{R})$,

$$
f\left(B_{t}^{H}\right)=f(0)+\int_{0}^{t} f^{\prime}\left(B_{u}^{H}\right) d^{\circ} B_{u}^{H} .
$$

This explains why $H=\frac{1}{6}$ is a natural barrier for the validity of Itô-Stratonovich formula. Also it is the sharp extension of the result of [15] obtained for $H \geqslant \frac{1}{4}$.

2. An example of probability measure satisfying (4.4) is given by

$$
v=\sum_{j=0}^{2(r-1)} \gamma_{j} \delta_{j /(2 r-2)}, \quad \text { with } \gamma_{j}=\int_{0}^{1} \prod_{k \neq j} \frac{2(r-1) u-k}{j-k} d u
$$

Indeed, we can write, thanks to Newton-Cotes formula, see also [25, p. 118]:

$$
P(1)=P(0)+\int_{0}^{1} P^{\prime}(x) v(d x), \quad \text { with } P \in \mathbb{R}_{2 r-1}[X] .
$$

Choosing $P(X)=X^{2 j+1}$ for $j \in\{1, \ldots, r-1\}$, we obtain $1=(2 j+1) m_{2 j}$.

3. For instance, for $r=2$, choosing $v=\frac{1}{6} \delta_{0}+\frac{2}{3} \delta_{1 / 2}+\frac{1}{6} \delta_{1}$, we obtain Itô's formula (4.5) for $H>\frac{1}{10}$ and $f \in \mathrm{C}^{10}(\mathbb{R})$.

Remark 4.6. As we said, fractional Brownian motion is a peculiar example of validity of Itô formula. In reality, extensions are possible in several directions.

- First of all, the result would remain valid for any Gaussian process having a similar covariance structure. Considering the technicality of a more general statement we have preferred to restrict it to fractional Brownian motion. 
- Another possible extension goes in the direction of perturbing the fractional Brownian motion $B^{H}$ with a finite variation process; typically one can imagine a fractional Brownian motion with drift $B_{t}^{H}+\int_{0}^{t} u_{s} d s$, where $\left(u_{s}\right)$ is a locally integrable process. As it has been observed in [19], Itô formula can be extended to processes of the type $f\left(B^{H}, V\right)$ where $V$ is a bounded variation process.

\section{Proof of Theorem 4.1}

Step 1: Proofs of easily deducible statements.

$N$ will denote a standard Gaussian random variable.

- (Proof of 1) In [14] it is proved that, if $C$ is a continuous process, as $\varepsilon \downarrow 0$,

$$
\int_{0}^{t} C_{u}\left(\frac{B_{u+\varepsilon}^{H}-B_{u}^{H}}{\varepsilon^{H}}\right)^{2 n} d u \rightarrow \mu_{2 n} \int_{0}^{t} C_{u} d u
$$

almost surely uniformly on each compact interval. Using this result, when $\varepsilon \downarrow 0$, we easily obtain

$$
\frac{1}{\varepsilon} \int_{0}^{t} d u\left(B_{u+\varepsilon}^{H}-B_{u}^{H}\right)^{2 n} \int_{0}^{1} g\left(B_{u}^{H}+\alpha\left(B_{u+\varepsilon}^{H}-B_{u}^{H}\right)\right) \nu(d \alpha) \sim \varepsilon^{2 n H-1} \mu_{2 n} \int_{0}^{t} g\left(B_{u}^{H}\right) d u,
$$

almost surely uniformly (in $t$ ) on each compact interval and the statements in the first part of Theorem 4.1 follow.

- (Proof of 2(b)) Let $m$ be an odd integer such that $m \geqslant 3$ and assume that $H=\frac{1}{2 m}$. Let also fix $t \geqslant 0$. [14] implies, when $\varepsilon \rightarrow 0$,

$$
\frac{1}{\varepsilon} \int_{0}^{t}\left(B_{u+\varepsilon}^{\frac{1}{2 m}}-B_{u}^{\frac{1}{2 m}}\right)^{m} d u=\frac{1}{\sqrt{\varepsilon}} \int_{0}^{t}\left(\frac{B_{u+\varepsilon}^{\frac{1}{2 m}}-B_{u}^{\frac{1}{2 m}}}{\varepsilon^{\frac{1}{2 m}}}\right)^{m} d u \stackrel{\text { (law) }}{\longrightarrow} \sqrt{c_{m, H} t} N
$$

see also Proposition 2.3 and Remark 2.4 in [15].

- (Proof of 2(c)) Assuming that $\frac{1}{\varepsilon} \int_{0}^{t}\left(B_{u+\varepsilon}^{H}-B_{u}^{H}\right)^{m} d u$ converges, as $\varepsilon \rightarrow 0$, toward a random variable $Z$ in probability, we deduce that

$$
\varepsilon^{\frac{1}{2}-m H} \frac{1}{\varepsilon} \int_{0}^{t}\left(B_{u+\varepsilon}^{H}-B_{u}^{H}\right)^{m} d u \stackrel{\text { (law) }}{\longrightarrow} 0, \quad \text { as } \varepsilon \rightarrow 0 .
$$

But this quantity equals $\frac{1}{\sqrt{\varepsilon}} \int_{0}^{t}\left(\frac{B_{u+\varepsilon}^{H}-B_{u}^{H}}{\varepsilon^{H}}\right)^{m} d u$ which, by [14], converges in law toward $\sqrt{c_{m, H} t} N$. This constitutes a contradiction.

- (Proof of 3) This point is a direct consequence of Proposition 3.3 and point 2(a).

We proceed now to the proof of point 2(a).

Step 2: First reduction.

- By a localization argument, see also the proof of Proposition 3.3, we can assume that $g$ and all its derivatives up to order $m$ are bounded.

- For simplicity, we fix $t=1$. 
- We can assume that $H \leqslant \frac{1}{m}<\frac{1}{2}$. Indeed, thanks to the following inequality

$$
\mathrm{E}\left|\frac{1}{\varepsilon} \int_{0}^{1} g\left(\frac{B_{u}^{H}+B_{u+\varepsilon}^{H}}{2}\right)\left(B_{u+\varepsilon}^{H}-B_{u}^{H}\right)^{m} d u\right| \leqslant \frac{\mathrm{cst} .}{\varepsilon} \int_{0}^{1} \mathrm{E}\left|B_{u+\varepsilon}^{H}-B_{u}^{H}\right|^{m} d u=\text { cst. } \varepsilon^{m H-1},
$$

we can easily show that integral $\int_{0}^{1} g\left(B_{u}^{H}\right) d^{\delta_{1 / 2}, m} B_{u}^{H}$ exists and vanishes, if $H>\frac{1}{m}$.

- Therefore, it suffices to prove the following.

Lemma 5.1. Let $m \geqslant 3$ be an odd integer. If $g: \mathbb{R} \rightarrow \mathbb{R}$ is a bounded function, then integral $\int_{0}^{1} g\left(B_{u}^{H}\right) d^{\delta_{1 / 2}, m} B_{u}^{H}$ exist and vanish for $\frac{1}{2 m}<H \leqslant \frac{1}{m}<\frac{1}{2}$.

- In proving Lemma 5.1 we need to consider

$$
J_{\varepsilon}^{(m)}(g):=\frac{1}{\varepsilon} \int_{0}^{1} g\left(\frac{B_{u+\varepsilon}^{H}+B_{u}^{H}}{2}\right)\left(B_{u+\varepsilon}^{H}-B_{u}^{H}\right)^{m} d u
$$

and to prove that

$$
\begin{aligned}
& E\left\{J_{\varepsilon}^{(m)}(g)^{2}\right\} \\
& =\frac{1}{2 \varepsilon^{2}} \iint_{D_{0}} E\left\{g\left(\frac{B_{u+\varepsilon}^{H}+B_{u}^{H}}{2}\right) g\left(\frac{B_{v+\varepsilon}^{H}+B_{v}^{H}}{2}\right)\left(B_{u+\varepsilon}^{H}-B_{u}^{H}\right)^{m}\left(B_{v+\varepsilon}^{H}-B_{v}^{H}\right)^{m}\right\} d u d v
\end{aligned}
$$

tends to zero as $\varepsilon \downarrow 0$. Here $D_{0}:=\{0<u<v<1\}$.

- It suffices to analyze the integral in (5.1) only on

$$
D_{\varepsilon}:=\left\{\varepsilon^{1-\rho}<u<v<1, \varepsilon^{1-\rho}<v-u<1\right\}, \quad \text { with } \rho>0 \text { small enough. }
$$

Indeed, using the hypothesis on $g$, the absolute value of

$$
\mathcal{J}^{\prime}(\varepsilon):=\frac{1}{2 \varepsilon^{2}} \iint_{D_{0} \backslash D_{\varepsilon}} E\left\{g\left(\frac{B_{u+\varepsilon}^{H}+B_{u}^{H}}{2}\right) g\left(\frac{B_{v+\varepsilon}^{H}+B_{v}^{H}}{2}\right)\left(B_{u+\varepsilon}^{H}-B_{u}^{H}\right)^{m}\left(B_{v+\varepsilon}^{H}-B_{v}^{H}\right)^{m}\right\} d u d v
$$

can be bounded by

$$
\frac{\text { cst. }}{\varepsilon^{2}} \underset{D_{0} \backslash D_{\varepsilon}}{\int} E\left[\left|B_{u+\varepsilon}^{H}-B_{u}^{H}\right|^{m}\left|B_{v+\varepsilon}^{H}-B_{v}^{H}\right|^{m}\right] d u d v \leqslant \operatorname{cst} . \varepsilon^{2 m H-2} \operatorname{mes}\left(D_{0} \backslash D_{\varepsilon}\right) \leqslant \operatorname{cst} . \varepsilon^{2 m H-1-\rho} .
$$

Choosing $0<\rho<2 m H-1$ we can see that $\mathcal{J}^{\prime}(\varepsilon)$ converges to 0 , as $\varepsilon \downarrow 0$. Hence it is sufficient to prove that

$$
\mathcal{J}(\varepsilon):=\frac{1}{2 \varepsilon^{2}} \iint_{D_{\varepsilon}} E\left\{g\left(\frac{B_{u+\varepsilon}^{H}+B_{u}^{H}}{2}\right) g\left(\frac{B_{v+\varepsilon}^{H}+B_{v}^{H}}{2}\right)\left(B_{u+\varepsilon}^{H}-B_{u}^{H}\right)^{m}\left(B_{v+\varepsilon}^{H}-B_{v}^{H}\right)^{m}\right\} d u d v
$$

tends to zero as $\varepsilon \downarrow 0$.

Step 3: Linear regression.

For notational convenience, we drop the index $H$ in $B^{H}$. Let us fix $\varepsilon>0$ and $(u, v) \in D_{\varepsilon}$. We will operate Gaussian analysis on the mean zero Gaussian vector $\left(G_{1}, G_{2}, G_{3}, G_{4}\right)$ where

$$
\left(G_{1}, G_{2}, G_{3}, G_{4}\right):=\left(B_{u+\varepsilon}+B_{u}, B_{v+\varepsilon}+B_{v}, B_{u+\varepsilon}-B_{u}, B_{v+\varepsilon}-B_{v}\right) .
$$


Its covariance matrix is given using blocks by

$$
\Lambda=\left(\begin{array}{ll}
\Lambda_{11} & \Lambda_{21}^{\star} \\
\Lambda_{21} & \Lambda_{22}
\end{array}\right),
$$

where $\Lambda_{11}$ (resp. $\left.\Lambda_{22}\right)$ is the covariance matrix of $\left(G_{1}, G_{2}\right)$ (resp. $\left.\left(G_{3}, G_{4}\right)\right)$ and

$$
\Lambda_{21}=\left(\begin{array}{ll}
\operatorname{Cov}\left(G_{3}, G_{1}\right) & \operatorname{Cov}\left(G_{3}, G_{2}\right) \\
\operatorname{Cov}\left(G_{4}, G_{1}\right) & \operatorname{Cov}\left(G_{4}, G_{2}\right)
\end{array}\right)
$$

Classical linear regression says that

$$
\left(\begin{array}{l}
G_{3} \\
G_{4}
\end{array}\right)=A\left(\begin{array}{l}
G_{1} \\
G_{2}
\end{array}\right)+\left(\begin{array}{c}
Z_{3} \\
Z_{4}
\end{array}\right)
$$

where $\left(Z_{3}, Z_{4}\right)$ is a mean zero Gaussian random vector independent from $\left(G_{1}, G_{2}\right)$ and

$$
A=\Lambda_{21} \Lambda_{11}^{-1} \text {. }
$$

We have

$$
\Lambda_{11}=2\left(\begin{array}{ll}
K^{\varepsilon}(u, u) & K^{\varepsilon}(u, v) \\
K^{\varepsilon}(u, v) & K^{\varepsilon}(v, v)
\end{array}\right)
$$

with

$$
\begin{aligned}
K^{\varepsilon}(u, v):= & \frac{1}{2}\left((u+\varepsilon)^{2 H}+(v+\varepsilon)^{2 H}+u^{2 H}+v^{2 H}-|v-u|^{2 H}\right. \\
& \left.-\frac{1}{2}|v-u-\varepsilon|^{2 H}-\frac{1}{2}|v-u+\varepsilon|^{2 H}\right) .
\end{aligned}
$$

Note that $\lim _{\varepsilon \rightarrow 0} K^{\varepsilon}(u, v)=K_{H}(u, v)$, the covariance of $B_{u}$ and $B_{v}$. We have

$$
\Lambda_{21}=\left(\begin{array}{cc}
\alpha(\varepsilon, u) & \alpha(\varepsilon, u)+\frac{1}{2} \alpha(\varepsilon, v-u)-\frac{1}{2} \alpha(-\varepsilon, v-u) \\
\alpha(\varepsilon, v)+\frac{1}{2} \alpha(\varepsilon, v-u)-\frac{1}{2} \alpha(-\varepsilon, v-u) & \alpha(\varepsilon, v)
\end{array}\right),
$$

with

$$
\alpha(\varepsilon, u)=(u+\varepsilon)^{2 H}-u^{2 H}=\varepsilon u^{2 H-1} \Psi\left(\frac{\varepsilon}{u}\right),
$$

where $\Psi: \mathbb{R} \rightarrow \mathbb{R}$ is a bounded function, defined by $\Psi(x)=\frac{(1+x)^{2 H}-1}{x}$. Since $\Lambda_{11}$ is a symmetric positive matrix, we can express $\Lambda_{11}=M M^{\star}, M$ being the Cholesky matrix of $\Lambda_{11}^{x}$, that is

$$
M:=\sqrt{2}\left(\begin{array}{cc}
\sqrt{K^{\varepsilon}(u, u)} & 0 \\
\frac{K^{\varepsilon}(u, v)}{\sqrt{K^{\varepsilon}(u, u)}} & \sqrt{K^{\varepsilon}(v, v)-\frac{K^{\varepsilon}(u, v)^{2}}{K^{\varepsilon}(u, u)}}
\end{array}\right) .
$$

Then, if we define the mean zero Gaussian vector $\left(N_{1}, N_{2}\right)$ by

$$
\left(\begin{array}{l}
G_{1} \\
G_{2}
\end{array}\right)=M\left(\begin{array}{l}
N_{1} \\
N_{2}
\end{array}\right)
$$

the random variables $N_{1}$ and $N_{2}$ are independent and we have

$$
\left(\begin{array}{l}
G_{3} \\
G_{4}
\end{array}\right)=R\left(\begin{array}{l}
N_{1} \\
N_{2}
\end{array}\right)+\left(\begin{array}{l}
Z_{3} \\
Z_{4}
\end{array}\right)
$$

with $R=\Lambda_{21} M^{\star-1}=\left\{r_{i j}\right\}_{1 \leqslant i, j \leqslant 2}$. For convenience we set

$$
\left(\begin{array}{l}
\Gamma_{3} \\
\Gamma_{4}
\end{array}\right):=A\left(\begin{array}{l}
G_{1} \\
G_{2}
\end{array}\right)=R\left(\begin{array}{l}
N_{1} \\
N_{2}
\end{array}\right)=\left(\begin{array}{l}
r_{11} N_{1}+r_{12} N_{2} \\
r_{21} N_{1}+r_{22} N_{2}
\end{array}\right) .
$$


Step 4: Splitting $\mathcal{J}(\varepsilon)$ in three terms.

We compute

$$
\begin{aligned}
\mathcal{J}(\varepsilon) & =\frac{1}{2 \varepsilon^{2}} \iint_{D_{\varepsilon}} E\left\{g\left(\frac{G_{1}}{2}\right) g\left(\frac{G_{2}}{2}\right) G_{3}^{m} G_{4}^{m}\right\} d u d v \\
& =\frac{1}{2 \varepsilon^{2}} \iint_{D_{\varepsilon}} E\left\{g\left(\frac{G_{1}}{2}\right) g\left(\frac{G_{2}}{2}\right)\left(\Gamma_{3}+Z_{3}\right)^{m}\left(\Gamma_{4}+Z_{4}\right)^{m}\right\} d u d v=\mathcal{J}_{1}(\varepsilon)+\mathcal{J}_{2}(\varepsilon)+\mathcal{J}_{3}(\varepsilon),
\end{aligned}
$$

where

$$
\begin{aligned}
& \mathcal{J}_{1}(\varepsilon):=\frac{1}{2 \varepsilon^{2}} \iint_{D_{\varepsilon}} E\left\{g\left(\frac{G_{1}}{2}\right) g\left(\frac{G_{2}}{2}\right) Z_{3}^{m} Z_{4}^{m}\right\} d u d v, \\
& \mathcal{J}_{2}(\varepsilon):=\frac{m}{2 \varepsilon^{2}} \iint_{D_{\varepsilon}} E\left\{g\left(\frac{G_{1}}{2}\right) g\left(\frac{G_{2}}{2}\right)\left(\Gamma_{3} Z_{3}^{m-1} Z_{4}^{m}+\Gamma_{4} Z_{3}^{m} Z_{4}^{m-1}\right)\right\} d u d v
\end{aligned}
$$

and

$$
\mathcal{J}_{3}(\varepsilon):=\frac{1}{2 \varepsilon^{2}} \iint_{D_{\varepsilon}} E\left\{g\left(\frac{G_{1}}{2}\right) g\left(\frac{G_{2}}{2}\right) \sum_{j=0}^{m} \sum_{k=2}^{m} C_{m}^{j} C_{m}^{k}\left(\Gamma_{3}^{j} Z_{3}^{m-j} \Gamma_{4}^{k} Z_{4}^{m-k}+\Gamma_{3}^{k} Z_{3}^{m-k} \Gamma_{4}^{j} Z_{4}^{m-j}\right)\right\} d u d v .
$$

- We remark that $\mathcal{J}_{2}(\varepsilon)=0$. Indeed, by the independence of $\left(G_{1}, G_{2}\right)$ and $\left(Z_{3}, Z_{4}\right)$, we can write

$$
\begin{aligned}
& E\left\{g\left(\frac{G_{1}}{2}\right) g\left(\frac{G_{2}}{2}\right)\left(\Gamma_{3} Z_{3}^{m-1} Z_{4}^{m}+\Gamma_{4} Z_{3}^{m} Z_{4}^{m-1}\right)\right\} \\
& =E\left\{g\left(\frac{G_{1}}{2}\right) g\left(\frac{G_{2}}{2}\right) \Gamma_{3}\right\} E\left\{Z_{3}^{m-1} Z_{4}^{m}\right\}+E\left\{g\left(\frac{G_{1}}{2}\right) g\left(\frac{G_{2}}{2}\right) \Gamma_{4}\right\} E\left\{Z_{3}^{m} Z_{4}^{m-1}\right\}=0,
\end{aligned}
$$

as we obtain by the first part of the following result.

Lemma 5.2. Let $\left(Z_{3}, Z_{4}\right)$ be a centered Gaussian random vector and $m$ a positive integer. Then

$$
E\left\{Z_{3}^{m-1} Z_{4}^{m}\right\}=0
$$

and

$$
E\left\{Z_{3}^{m} Z_{4}^{m}\right\}=\sum_{j=0}^{\left[\frac{m-1}{2}\right]} c_{j} E\left\{Z_{3} Z_{4}\right\}^{m-2 j} \operatorname{Var}\left(Z_{3}\right)^{j} \operatorname{Var}\left(Z_{4}\right)^{j},
$$

with $c_{j}$ some universal constants.

The proof of this result is postponed to step 5 .

- We shall prove that each term in $\mathcal{J}_{3}(\varepsilon)$ tends to zero. (5.6) entails

$$
E\left\{Z_{\ell}^{2}\right\}=E\left\{G_{\ell}^{2}\right\}-E\left\{\Gamma_{\ell}^{2}\right\} \leqslant E\left\{G_{\ell}^{2}\right\}=\varepsilon^{2 H}, \quad \ell=3,4 .
$$

Let $j \neq 0$ and $k \geqslant 2$. Since $g$ is bounded,

$$
\frac{1}{2 \varepsilon^{2}} \iint_{D_{\varepsilon}}\left|E\left\{g\left(\frac{G_{1}}{2}\right) g\left(\frac{G_{2}}{2}\right) \Gamma_{3}^{j} Z_{3}^{m-j} \Gamma_{4}^{k} Z_{4}^{m-k}\right\}\right| d u d v \leqslant \frac{\mathrm{cst} .}{\varepsilon^{2}} \iint_{D_{\varepsilon}} E\left\{\left|\Gamma_{3}^{j} Z_{3}^{m-j} \Gamma_{4}^{k} Z_{4}^{m-k}\right|\right\} d u d v
$$


by Cauchy-Schwarz inequality, previous term is bounded by

$$
\frac{\text { cst. }}{\varepsilon^{2}} \iint_{D_{\varepsilon}}\left[E\left\{\Gamma_{3}^{2 j} Z_{3}^{2 m-2 j}\right\}\right]^{\frac{1}{2}}\left[E\left\{\Gamma_{4}^{2 k} Z_{4}^{2 m-2 k}\right\}\right]^{\frac{1}{2}} d u d v ;
$$

using the independence of $\left(G_{1}, G_{2}\right)$ and $\left(Z_{3}, Z_{4}\right)$, it equals

$$
\frac{\text { cst. }}{\varepsilon^{2}} \iint_{D_{\varepsilon}}\left[E\left\{\Gamma_{3}^{2 j}\right\} E\left\{Z_{3}^{2 m-2 j}\right\}\right]^{\frac{1}{2}}\left[E\left\{\Gamma_{4}^{2 k}\right\} E\left\{Z_{4}^{2 m-2 k}\right\}\right]^{\frac{1}{2}} d u d v
$$

again by Cauchy-Schwarz inequality, this is less or equal than

$$
\frac{\text { cst. }}{\varepsilon^{2}}\left(\iint_{D_{\varepsilon}} E\left\{\Gamma_{3}^{2 j}\right\} E\left\{Z_{3}^{2 m-2 j}\right\} d u d v\right)^{\frac{1}{2}}\left(\iint_{D_{\varepsilon}} E\left\{\Gamma_{4}^{2 k}\right\} E\left\{Z_{4}^{2 m-2 k}\right\} d u d v\right)^{\frac{1}{2}} .
$$

Using (5.9) and Lemma 5.3 we obtain the following bound:

$$
\text { cst. } \varepsilon^{(2 m-j-k) H-2}\left(\iint_{D_{\varepsilon}} E\left\{\Gamma_{3}^{2 j}\right\} d u d v\right)^{\frac{1}{2}}\left(\iint_{D_{\varepsilon}} E\left\{\Gamma_{4}^{2 k}\right\} d u d v\right)^{\frac{1}{2}} \leqslant \text { cst. } \varepsilon^{2 m H-1} \text {. }
$$

This converges to zero because $H>\frac{1}{2 m}$. The last inequality is a consequence of the following technical result which proof is postponed to step 5 .

Lemma 5.3. Let $k \geqslant 2$ be a integer. Then

$$
\iint_{D_{\varepsilon}} E\left\{\left|\Gamma_{\ell}\right|^{k}\right\} d u d v \leqslant \text { cst. } \varepsilon^{1+k H}, \quad \ell=3,4 .
$$

If $j=0$, and $k \geqslant 2$,

$$
\begin{aligned}
& \frac{1}{2 \varepsilon^{2}} \iint_{D_{\varepsilon}}\left|E\left\{g\left(\frac{G_{1}}{2}\right) g\left(\frac{G_{2}}{2}\right) Z_{3}^{m} \Gamma_{4}^{k} Z_{4}^{m-k}\right\}\right| d u d v \\
& \leqslant \frac{\text { cst. }}{\varepsilon^{2}} \iint_{D_{\varepsilon}} E\left\{\left|Z_{3}^{m} \Gamma_{4}^{k} Z_{4}^{m-k}\right|\right\} d u d v=\frac{\text { cst. }}{\varepsilon^{2}} \iint_{D_{\varepsilon}} E\left\{\left|\Gamma_{4}\right|^{k}\right\} E\left\{\left|Z_{3}^{m} Z_{4}^{m-k}\right|\right\} d u d v \\
& \leqslant \frac{\text { cst. }}{\varepsilon^{2}} \iint_{D_{\varepsilon}}\left[E\left\{Z_{3}^{2 m}\right\}\right]^{\frac{1}{2}}\left[E\left\{Z_{4}^{2 m-2 k}\right\}\right]^{\frac{1}{2}} E\left\{\left|\Gamma_{4}\right|^{k}\right\} d u d v \\
& \leqslant \text { cst. } \varepsilon^{(2 m-k) H-2} \iint_{D_{\varepsilon}} E\left\{\left|\Gamma_{4}\right|^{k}\right\} d u d v \leqslant \text { cst. } \varepsilon^{2 m H-1} .
\end{aligned}
$$

Therefore, $\lim _{\varepsilon \downarrow 0} \mathcal{J}_{3}(\varepsilon)=0$.

- We need to prove that $\lim _{\varepsilon \downarrow 0} \mathcal{J}_{1}(\varepsilon)=0$. By independence of $\left(G_{1}, G_{2}\right)$ and $\left(Z_{3}, Z_{4}\right)$, we can write

$$
\mathcal{J}_{1}(\varepsilon)=\frac{1}{2 \varepsilon^{2}} \iint_{D_{\varepsilon}} E\left\{g\left(\frac{G_{1}}{2}\right) g\left(\frac{G_{2}}{2}\right)\right\} E\left\{Z_{3}^{m} Z_{4}^{m}\right\} d u d v
$$


and, since $g$ is bounded,

$$
\left|\mathcal{J}_{1}(\varepsilon)\right| \leqslant \frac{\text { cst. }}{\varepsilon^{2}} \iint_{D_{\varepsilon}}\left|E\left\{Z_{3}^{m} Z_{4}^{m}\right\}\right| d u d v .
$$

We state the following result which will be proved in step 5 .

Lemma 5.4. For every $j \in\left\{0, \ldots, \frac{m-1}{2}\right\}$, we have

$$
\iint_{D_{\varepsilon}}\left|E\left\{Z_{3} Z_{4}\right\}\right|^{m-2 j} d u d v \leqslant \text { cst. } \varepsilon^{1+2(m-2 j) H} .
$$

Now, we can show that $\mathcal{J}_{1}(\varepsilon)$ tends to zero as $\varepsilon \downarrow 0$ as follows. We know that $\operatorname{Var}\left(Z_{i}\right) \leqslant \varepsilon^{2 H}, i=3,4$; by using (5.8) and Lemma 5.4, we can write

$$
\begin{aligned}
\left|\mathcal{J}_{1}(\varepsilon)\right| & \leqslant \frac{\text { cst. }}{\varepsilon^{2}} \sum_{j=0}^{\frac{m-1}{2}} \int_{2 \varepsilon}^{1} d v \int_{\varepsilon}^{v-\varepsilon} d u\left|E\left\{Z_{3} Z_{4}\right\}\right|^{m-2 j} \operatorname{Var}\left(Z_{3}\right)^{j} \operatorname{Var}\left(Z_{4}\right)^{j} \\
& \leqslant \frac{\text { cst. }}{\varepsilon^{2}} \sum_{j=0}^{\frac{m-1}{2}} \varepsilon^{4 j H} \int_{2 \varepsilon}^{1} d v \int_{\varepsilon}^{v-\varepsilon} d u\left|E\left\{Z_{3} Z_{4}\right\}\right|^{m-2 j} \leqslant \text { cst. } \varepsilon^{2 m H-1}
\end{aligned}
$$

and $\mathcal{J}_{1}(\varepsilon)$ tends to zero as $\varepsilon \rightarrow 0$ since $H>\frac{1}{2 m}$.

Step 5: Proofs of Lemmas 5.2, 5.3 and 5.4.

\section{Proof of Lemma 5.2.}

(i) (5.7) is obvious because the random vectors $\left(Z_{3}, Z_{4}\right)$ and $\left(-Z_{3},-Z_{4}\right)$ have the same distribution.

(ii) We denote $\theta=E\left\{Z_{3} Z_{4}\right\}$ and $\sigma_{i}^{2}=\operatorname{Var}\left(Z_{i}\right), i=3$, 4. By linear regression, we can write

$$
Z_{4}=\frac{\theta}{\sigma_{3}^{2}} Z_{3}+\left(\frac{\sigma_{3}^{2} \sigma_{4}^{2}-\theta^{2}}{\sigma_{3}^{2}}\right)^{\frac{1}{2}} N
$$

with $N$ a standard Gaussian variable independent of $Z_{3}$. We denote $\mu_{2 k}$ the (2k)-moment of $N$. We have

$$
\begin{aligned}
E\left\{Z_{3}^{m} Z_{4}^{m}\right\} & =\sum_{k=0}^{\left[\frac{m-1}{2}\right]} C_{m}^{2 k}\left(\frac{\theta}{\sigma_{3}^{2}}\right)^{m-2 k} E\left\{Z_{3}^{2(m-k)}\right\}\left(\frac{\sigma_{3}^{2} \sigma_{4}^{2}-\theta^{2}}{\sigma_{3}^{2}}\right)^{k} \mu_{2 k} \\
& =\sum_{k=0}^{\left[\frac{m-1}{2}\right]} \mu_{2 k} \mu_{2(m-k)} C_{m}^{2 k} \theta^{m-2 k} \sum_{\ell=0}^{k}(-1)^{\ell} C_{k}^{\ell} \theta^{2 \ell} \sigma_{3}^{2(k-\ell)} \sigma_{4}^{2(k-\ell)} \\
& =\sum_{k=0}^{\left.\frac{m-1}{2}\right]} \sum_{\ell=0}^{k} c_{k, \ell} \theta^{m-2(k-\ell)} \sigma_{3}^{2(k-\ell)} \sigma_{4}^{2(k-\ell)} \\
& =\sum_{j=0}^{\left.\frac{m-1}{2}\right]} c_{j} \theta^{m-2 j} \sigma_{3}^{2 j} \sigma_{4}^{2 j} .
\end{aligned}
$$


Proof of Lemma 5.3. We have, by (5.6)

$$
\begin{aligned}
E\left\{\iint_{D_{\varepsilon}}\left|\Gamma_{3}\right|^{k} d u d v\right\} & \leqslant \operatorname{cst} .\left(\iint_{D_{\varepsilon}}\left|r_{11}\right|^{k} E\left\{\left|N_{1}\right|^{k}\right\} d u d v+\iint_{D_{\varepsilon}}\left|r_{12}\right|^{k} E\left\{\left|N_{2}\right|^{k}\right\} d u d v\right) \\
& =\operatorname{cst} .\left(\iint_{D_{\varepsilon}}\left|r_{11}\right|^{k} d u d v+\iint_{D_{\varepsilon}}\left|r_{12}\right|^{k} d u d v\right)
\end{aligned}
$$

and

$$
\begin{aligned}
E\left\{\iint_{D_{\varepsilon}}\left|\Gamma_{4}\right|^{k} d u d v\right\} & \leqslant \operatorname{cst}\left(\iint_{D_{\varepsilon}}\left|r_{21}\right|^{k} E\left\{\left|N_{1}\right|^{k}\right\} d u d v+\iint_{D_{\varepsilon}}\left|r_{22}\right|^{k} E\left\{\left|N_{2}\right|^{k}\right\} d u d v\right) \\
& =\operatorname{cst}\left(\iint_{D_{\varepsilon}}\left|r_{21}\right|^{k} d u d v+\iint_{D_{\varepsilon}}\left|r_{22}\right|^{k} d u d v\right) .
\end{aligned}
$$

The proof will be done once we show

$$
\iint_{D_{\varepsilon}}\left|r_{i j}\right|^{k} d u d v \leqslant \operatorname{cst} . \varepsilon^{1+k H}
$$

for all $i, j \in\{1,2\}$. Recall that

$$
R=\Lambda_{21} M^{\star-1}=\left(\begin{array}{ll}
r_{11} & r_{12} \\
r_{21} & r_{22}
\end{array}\right)
$$

with

$$
M^{\star-1}=\frac{1}{\sqrt{2}}\left(\begin{array}{cc}
\frac{1}{\sqrt{K^{\varepsilon}(u, u)}} & -\frac{K^{\varepsilon}(u, v)}{\sqrt{K^{\varepsilon}(u, u) \Delta^{\varepsilon}(u, v)}} \\
0 & \sqrt{\frac{K^{\varepsilon}(u, u)}{\Delta^{\varepsilon}(u, v)}}
\end{array}\right),
$$

and

$$
\Delta^{\varepsilon}(u, v):=K^{\varepsilon}(u, u) K^{\varepsilon}(v, v)-K^{\varepsilon}(u, v)^{2}, \quad \text { with } K^{\varepsilon}(u, v) \text { given by (5.3). }
$$

Moreover, by (5.4),

$$
\begin{aligned}
& \Lambda_{21}[1,1]=\varepsilon u^{2 H-1} \Psi\left(\frac{\varepsilon}{u}\right), \\
& \Lambda_{21}[1,2]=\varepsilon u^{2 H-1} \Psi\left(\frac{\varepsilon}{u}\right)+\frac{\varepsilon}{2}(v-u)^{2 H-1} \Psi\left(\frac{\varepsilon}{v-u}\right)+\frac{\varepsilon}{2}(v-u)^{2 H-1} \Psi\left(-\frac{\varepsilon}{v-u}\right), \\
& \Lambda_{21}[2,1]=\varepsilon v^{2 H-1} \Psi\left(\frac{\varepsilon}{v}\right)+\frac{\varepsilon}{2}(v-u)^{2 H-1} \Psi\left(\frac{\varepsilon}{v-u}\right)+\frac{\varepsilon}{2}(v-u)^{2 H-1} \Psi\left(-\frac{\varepsilon}{v-u}\right), \\
& \Lambda_{21}[2,2]=\varepsilon v^{2 H-1} \Psi\left(\frac{\varepsilon}{v}\right) .
\end{aligned}
$$

At this point we need to establish some useful estimates.

Lemma 5.5. There exist positive constants $c_{1}$ and $c_{2}$ such that, for any $\varepsilon>0$ and any $(u, v) \in D_{\varepsilon}$, we have

$$
\begin{aligned}
& c_{1} u^{2 H} \leqslant K^{\varepsilon}(u, u) \leqslant c_{2} u^{2 H}, \quad K^{\varepsilon}(u, v) \leqslant c_{2} u^{H} v^{H}, \\
& \Delta^{\varepsilon}(u, v) \geqslant c_{1} u^{2 H}(v-u)^{2 H} .
\end{aligned}
$$


Thanks to Lemma 5.5 and boundedness of $\Psi$, we deduce

$$
\begin{aligned}
& \left|r_{11}\right| \leqslant \operatorname{cst} . \varepsilon u^{H-1}, \\
& \left|r_{21}\right| \leqslant \operatorname{cst} . \varepsilon\left(\frac{v^{2 H-1}}{u^{H}}+\frac{(v-u)^{2 H-1}}{u^{H}}\right), \\
& \left|r_{12}\right| \leqslant \operatorname{cst} . \varepsilon\left(\frac{u^{H-1} v^{H}}{(v-u)^{H}}+\frac{u^{2 H-1}}{(v-u)^{H}}+(v-u)^{H-1}\right), \\
& \left|r_{22}\right| \leqslant \operatorname{cst} . \varepsilon\left(\frac{v^{3 H-1}}{u^{H}(v-u)^{H}}+\frac{v^{2 H-1}}{(v-u)^{H}}+\frac{v^{H}(v-u)^{H-1}}{u^{H}}\right) .
\end{aligned}
$$

(5.10) will be now a consequence of last four inequalities. This ends the proof of Lemma 5.3 excepted for the proof of Lemma 5.5.

Proof of Lemma 5.5. Since $0<2 H<1$, we have, for any $x, y>0$

$$
(x+y)^{2 H} \geqslant 2^{2 H-1}\left(x^{2 H}+y^{2 H}\right) \geqslant \frac{1}{2}\left(x^{2 H}+y^{2 H}\right) .
$$

We deduce

$$
K^{\varepsilon}(u, u)=(u+\varepsilon)^{2 H}+u^{2 H}-\frac{\varepsilon^{2 H}}{2} \geqslant \frac{3}{2} u^{2 H}
$$

and

$$
K^{\varepsilon}(u, u) \leqslant 2\left(2^{H}+1\right) u^{2 H},
$$

since $u>\varepsilon^{1-\rho}>\varepsilon$. By Cauchy-Schwarz inequality, we have

$$
2 K^{\varepsilon}(u, v)=E\left\{G_{1} G_{2}\right\} \leqslant \operatorname{Var}\left(\mathrm{G}_{1}\right)^{\frac{1}{2}} \operatorname{Var}\left(\mathrm{G}_{2}\right)^{\frac{1}{2}}=2 K^{\varepsilon}(u, u)^{\frac{1}{2}} K^{\varepsilon}(v, v)^{\frac{1}{2}} \leqslant 4\left(2^{H}+1\right) u^{H} v^{H} .
$$

It remains to justify the lower bound for $\Delta^{\varepsilon}$. We set

$$
\frac{\Delta^{\varepsilon}(u, v)}{u^{2 H}(v-u)^{2 H}}=\varphi\left(\frac{\varepsilon}{u}, \frac{v}{u}\right)
$$

with $\varphi:[0,1] \times] 1,+\infty[$ defined by

$$
\begin{aligned}
4 \varphi(\delta, x):= & \frac{x^{2 H}(x-1-\delta)^{2 H}}{(x-1)^{2 H}}+2 \frac{(1+\delta)^{2 H} x^{2 H}}{(x-1)^{2 H}}+2 x^{2 H}+2(1+\delta)^{2 H}+2(x+\delta)^{2 H}-\frac{1}{(x-1)^{2 H}} \\
& -(x-1)^{2 H}-\frac{x^{4 H}}{(x-1)^{2 H}}+\frac{(1+\delta)^{2 H}(x-1+\delta)^{2 H}}{(x-1)^{2 H}}-\frac{1}{4} \frac{(x-1+\delta)^{4 H}}{(x-1)^{2 H}}-2 \frac{\delta^{2 H}(x+\delta)^{2 H}}{(x-1)^{2 H}} \\
& -\frac{1}{2} \frac{(x-1-\delta)^{2 H}(x-1+\delta)^{2 H}}{(x-1)^{2 H}}-2 \frac{(1+\delta)^{2 H} \delta^{2 H}}{(x-1)^{2 H}}-(x-1+\delta)^{2 H}-(x-1-\delta)^{2 H} \\
& -2 \frac{(1+\delta)^{2 H}}{(x-1)^{2 H}}+\frac{x^{2 H}(x-1+\delta)^{2 H}}{(x-1)^{2 H}}+\frac{(x+\delta)^{2 H}(x-1-\delta)^{2 H}}{(x-1)^{2 H}}-2 \frac{(x+\delta)^{2 H} x^{2 H}}{(x-1)^{2 H}} \\
& -2 \frac{\delta^{2 H} x^{2 H}}{(x-1)^{2 H}}+\frac{(x+\delta)^{2 H}(x-1+\delta)^{2 H}}{(x-1)^{2 H}}-\frac{(1+\delta)^{4 H}}{(x-1)^{2 H}}+\frac{(1+\delta)^{2 H}(x-1-\delta)^{2 H}}{(x-1)^{2 H}} \\
& +\frac{(x-1-\delta)^{2 H}}{(x-1)^{2 H}}+2-\frac{1}{4} \frac{(x-1-\delta)^{4 H}}{(x-1)^{2 H}}+2 \frac{(x+\delta)^{2 H}}{(x-1)^{2 H}}-2 \frac{\delta^{2 H}}{(x-1)^{2 H}}+\frac{(x-1+\delta)^{2 H}}{(x-1)^{2 H}} \\
& +\frac{\delta^{4 H}}{(x-1)^{2 H}}+2 \frac{(1+\delta)^{2 H}(x+\delta)^{2 H}}{(x-1)^{2 H}}+2 \frac{x^{2 H}}{(x-1)^{2 H}}-\frac{(x+\delta)^{4 H}}{(x-1)^{2 H}} .
\end{aligned}
$$


We remark that

$$
4 \varphi(0, x)=2\left(\frac{x}{x-1}\right)^{2 H}-\left(\frac{1}{x-1}\right)^{2 H}-(x-1)^{2 H}+2+2 x^{2 H}-\left(\frac{x^{2}}{x-1}\right)^{2 H} .
$$

In fact, if $v>u, \varphi\left(0, \frac{v}{u}\right) u^{2 H}(v-u)^{2 H}=\Delta^{0}(u, v)$ is the determinant of the covariance matrix of $\left(B_{u}, B_{v}\right)$. Consequently

$$
\forall x>1, \quad \varphi(0, x)>0 .
$$

It is not difficult to see that

$$
\lim _{x \downarrow 1} \varphi(0, x)=\lim _{x \uparrow \infty} \varphi(0, x)=4 .
$$

We deduce that the infimum of $\varphi(0, \cdot)$ is reached and it is strictly positive. Precisely, there exists a constant $c_{1}>0$ such that, for all $v>u$

$$
\varphi\left(0, \frac{v}{u}\right) \geqslant 2 c_{1} \text {. }
$$

The lower bound (5.12) will be obtained by proving

$$
\exists c, \alpha>0, \forall \varepsilon>0, \forall(u, v) \in D_{\varepsilon}: \quad\left|\varphi\left(\frac{\varepsilon}{u}, \frac{v}{u}\right)-\varphi\left(0, \frac{v}{u}\right)\right| \leqslant c \varepsilon^{\alpha} .
$$

Indeed, if $\varepsilon>0$ is small enough and if $(u, v) \in D_{\varepsilon}$ then (5.12) holds. In order to show (5.17), we prove

$$
\exists c, \alpha>0, \forall \delta>0, \forall x \geqslant 1+\delta^{1-\rho}: \quad|\varphi(\delta, x)-\varphi(0, x)| \leqslant c \delta^{\alpha} .
$$

Indeed, if (5.18) holds we have, by putting $x=\frac{v}{u}$ and $\delta=\frac{\varepsilon}{u}$,

$$
\forall \varepsilon>0, \forall(u, v) \in D_{\varepsilon}:\left|\varphi\left(\frac{\varepsilon}{u}, \frac{v}{u}\right)-\varphi\left(0, \frac{v}{u}\right)\right| \leqslant c\left(\frac{\varepsilon}{u}\right)^{\alpha} \leqslant c \varepsilon^{\rho \alpha} .
$$

In order to show (5.18), we prove that each term in the definition of $\varphi(\delta, x)$ verifies inequalities of type (5.18). For instance, the first term of $\varphi(\delta, x)$ can be handled as follows:

$$
\begin{aligned}
\left|\frac{x^{2 H}(x-1-\delta)^{2 H}}{(x-1)^{2 H}}-\frac{x^{2 H}(x-1)^{2 H}}{(x-1)^{2 H}}\right| & \leqslant \frac{x^{2 H}}{(x-1)^{2 H}}\left[(x-1-\delta)^{2 H}-(x-1)^{2 H}\right] \\
& \leqslant \operatorname{cst} . \delta^{2 H} \frac{x^{2 H}}{(x-1)^{2 H}}=\operatorname{cst} . \delta^{2 H}\left(1+\frac{1}{x-1}\right)^{2 H} \\
& \leqslant \text { cst. }\left\{\begin{array}{ll}
\delta^{2 H}, & \text { if } x>2 \\
\frac{\delta^{2 H}}{\delta^{(1-\rho) 2 H}}, & \text { if } \delta^{1-\rho} \leqslant x-1 \leqslant 1
\end{array} \leqslant \text { cst. } \delta^{2 \rho H} .\right.
\end{aligned}
$$

In the previous third inequality we used the following fact

$$
y \geqslant \delta>0 \Rightarrow\left|(y \pm \delta)^{2 H}-y^{2 H}\right| \leqslant 2 H \delta^{2 H} .
$$

The proof of the Lemma 5.5 is now concluded.

Proof of Lemma 5.4. Since

$$
D_{\varepsilon} \subset\{\varepsilon<u<v<1, \varepsilon<v-u<1\}
$$

it suffices to prove that

$$
\int_{2 \varepsilon}^{1} d v \int_{\varepsilon}^{v-\varepsilon} d u\left|E\left\{Z_{3} Z_{4}\right\}\right|^{m-2 j} \leqslant \text { cst. } \varepsilon^{1+2(m-2 j) H} .
$$


Since $\left(G_{1}, G_{2}\right)$ and $\left(Z_{3}, Z_{4}\right)$ are independent, we have

$$
E\left\{G_{3} G_{4}\right\}=E\left\{\left(\Gamma_{3}+Z_{3}\right)\left(\Gamma_{4}+Z_{4}\right)\right\}=E\left\{\Gamma_{3} \Gamma_{4}\right\}+E\left\{Z_{3} Z_{4}\right\}
$$

and

$$
\left|E\left\{Z_{3} Z_{4}\right\}\right|^{m-2 j} \leqslant \operatorname{cst} .\left(\left|E\left\{G_{3} G_{4}\right\}\right|^{m-2 j}+\left|E\left\{\Gamma_{3} \Gamma_{4}\right\}\right|^{m-2 j}\right) .
$$

(i) We can write

$$
\begin{aligned}
& \int_{2 \varepsilon}^{1} d v \int_{\varepsilon}^{v-\varepsilon} d u\left|E\left\{\Gamma_{3} \Gamma_{4}\right\}\right|^{m-2 j} \\
& \leqslant \int_{2 \varepsilon}^{1} d v \int_{\varepsilon}^{v-\varepsilon} d u E\left\{\Gamma_{3}^{2}\right\}^{\frac{m-2 j}{2}} E\left\{\Gamma_{4}^{2}\right\}^{\frac{m-2 j}{2}} \\
& \leqslant\left(\int_{2 \varepsilon}^{1} d v \int_{\varepsilon}^{v-\varepsilon} d u E\left\{\Gamma_{3}^{2}\right\}^{m-2 j}\right)^{\frac{1}{2}}\left(\int_{2 \varepsilon}^{1} d v \int_{\varepsilon}^{v-\varepsilon} d u E\left\{\Gamma_{4}^{2}\right\}^{m-2 j}\right)^{\frac{1}{2}} \\
& \leqslant \operatorname{cst.}\left(\int_{2 \varepsilon}^{1} d v \int_{\varepsilon}^{v-\varepsilon} d u E\left\{\Gamma_{3}^{2(m-2 j)}\right\}\right)^{\frac{1}{2}}\left(\int_{2 \varepsilon}^{1} d v \int_{\varepsilon}^{v-\varepsilon} d u E\left\{\Gamma_{4}^{2(m-2 j)}\right\}\right)^{\frac{1}{2}} \leqslant \operatorname{cst} . \varepsilon^{1+2(m-2 j) H},
\end{aligned}
$$

where the last inequality is obtained using Lemma 5.3.

(ii) We have

$$
E\left\{G_{3} G_{4}\right\}=\frac{1}{2}(v-u+\varepsilon)^{2 H}+\frac{1}{2}(v-u-\varepsilon)^{2 H}-(v-u)^{2 H}=\varepsilon^{2}(v-u)^{2 H-2} \Phi\left(\frac{\varepsilon}{v-u}\right),
$$

where $\Phi: \mathbb{R} \rightarrow \mathbb{R}$ is a bounded function given by $\Phi(x)=\frac{(1+x)^{2 H}+(1-x)^{2 H}-2}{2 x^{2}}$. We deduce that

$$
\begin{aligned}
\int_{2 \varepsilon}^{1} d v \int_{\varepsilon}^{v-\varepsilon} d u\left|E\left\{G_{3} G_{4}\right\}\right|^{m-2 j} & \leqslant \operatorname{cst} . \varepsilon^{2(m-2 j)} \int_{2 \varepsilon}^{1} d v \int_{0}^{v-\varepsilon} d u(v-u)^{(2 H-2)(m-2 j)} \\
& \leqslant \operatorname{cst} . \varepsilon^{2(m-2 j)} \int_{2 \varepsilon}^{1} d v \int_{2 \varepsilon}^{1} d v \int_{\varepsilon}^{v} u^{(2 H-2)(m-2 j)} d u \\
& \leqslant \operatorname{cst} . \varepsilon^{2(m-2 j)} \int_{\varepsilon}^{1} u^{(2 H-2)(m-2 j)} d u \leqslant \operatorname{cst} .\left[\varepsilon^{1+2(m-2 j) H}+\varepsilon^{2(m-2 j)}\right] .
\end{aligned}
$$

Since $H<\frac{1}{2}$ and $m-2 j \geqslant 1$, we have $1+2 H(m-2 j) \leqslant 1+m-2 j \leqslant 2(m-2 j)$. Consequently,

$$
\int_{2 \varepsilon}^{1} d v \int_{\varepsilon}^{v-\varepsilon} d u\left|E\left\{G_{3} G_{4}\right\}\right|^{m-2 j} \leqslant \text { cst. } \varepsilon^{1+2(m-2 j) H} .
$$




\section{Acknowledgements}

We are grateful to the anonymous referee for careful reading of the original manuscript and for useful remarks.

\section{References}

[1] E. Alos, J.L. Léon, D. Nualart, Stratonovich calculus for fractional Brownian motion with Hurst parameter less than $\frac{1}{2}$, Taiwanese J. Math. 5 (2001) 609-632.

[2] C. Bender, An Itô formula for generalized functionals of a fractional Brownian motion with arbitrary Hurst parameter, Stochastic Process. Appl. 124 (1) (2003) 81-106.

[3] P. Carmona, L. Coutin, G. Monseny, Stochastic integration with respect to fractional Brownian motion, Ann. Inst. H. Poincaré Probab. Statist. 39 (1) (2003) 27-68.

[4] P. Cheridito, D. Nualart, Stochastic integral of divergence type with respect to fractional Brownian motion with Hurst parameter $H \in$ (0, $\left.\frac{1}{2}\right)$, Preprint, Barcelona, 2002.

[5] L. Coutin, Z. Qian, Stochastic analysis, rough path analysis and fractional Brownian motions, Probab. Theory Related Fields 122 (1) (2002) 108-140.

[6] L. Decreusefond, A.S. Ustunel, Stochastic analysis of the fractional Brownian motion, Potential Anal. 10 (1998) $177-214$.

[7] R.M. Dudley, R. Norvaisa, Differentiability of Six Operators on Nonsmooth Functions and $p$-variation, Lecture Notes in Math., vol. 1703, Springer-Verlag, 1999.

[8] M. Errami, F. Russo, Covariation de convolutions de martingales, C. R. Acad. Sci. Sér. 1326 (1998) 601-609.

[9] M. Errami, F. Russo, $n$-covariation and symmetric SDEs driven by finite cubic variation process, Stoch. Process. Appl. 104 (2) (2000) 259-299.

[10] D. Feyel, A. De La Pradelle, On fractional Brownian processes, Potential Anal. 10 (3) (1999) 273-288.

[11] H. Föllmer, Calcul d'Itô sans probabilités, in: Séminaire de Probabilités XV 1979/80, in: Lecture Notes in Math., vol. 850, Springer-Verlag, 1981, pp. 143-150.

[12] H. Föllmer, P. Protter, A.N. Shiryaev, Quadratic covariation and an extension of Itô's formula, Bernoulli 1 (1995) $149-169$.

[13] M. Fukushima, T.T. Oshima, Dirichlet Forms and Symmetric Markov Processes, Walter de Gruyter, 1994.

[14] M. Gradinaru, I. Nourdin, Approximation at first and second order of the $m$-variation of the fractional Brownian motion, Electron. J. Probab. 8 (2003) 1-26, Paper 18.

[15] M. Gradinaru, F. Russo, P. Vallois, Generalized covariations, local time and Stratonovich Itô's formula for fractional Brownian motion with Hurst index $H \geqslant \frac{1}{4}$, Ann. Probab. 31 (2003) 1772-1820.

[16] T.J. Lyons, Differential equations driven by rough signals, Rev. Mat. Iberoamericana 14 (2) (1998) 215-310.

[17] T.J. Lyons, W. Zheng, A crossing estimate for the canonical process on a Dirichlet space and tightness result, in: Colloque Paul Lévy sur les processus stochastiques, Astérisque 157-158 (1998) 249-271.

[18] S. Nakao, Stochastic calculus for continuous additive functionals of zero energy, Wahrs. Verw. Geb. 68 (1995) 557-578, (1985).

[19] I. Nourdin, PhD Thesis, Nancy, 2004.

[20] D. Nualart, The Malliavin Calculus and Related Topics, Springer-Verlag, 1995.

[21] N. Privault, C. Tudor, Skorohod and pathwise stochastic calculus with respect to a $L^{2}$ - process, Random Operators and Stochastic Equations 8 (3) (2000) 1-24.

[22] F. Russo, P. Vallois, The generalized covariation process and Itô formula, Stochastic Process. Appl. 59 (1995) 81-104.

[23] F. Russo, P. Vallois, Itô formula for $C^{1}$-functions of semimartingales, Probab. Theory Related. Fields 104 (1996) $27-41$.

[24] F. Russo, P. Vallois, Stochastic calculus with respect to a finite quadratic variation process, Stochastics and Stochastics Reports 70 (2000) $1-40$.

[25] J. Stoer, R. Bulirsch, R. Bartels, W. Gautschi, C. Witzgall, Introduction to Numerical Analysis, Springer-Verlag, 1983.

[26] G. Trutnau, Stochastic calculus of generalized Dirichlet forms and applications to stochastic differential equations in infinite dimensions, Osaka J. Math. 37 (2) (2000) 315-343.

[27] J. Wolf, An Itô Formula for local Dirichlet processes, Stochastics and Stochastics Reports 62 (2) (1997) $103-115$.

[28] M. Yor, Sur quelques approximations d'intégrales stochastiques, in: Séminaire de Probabilités XI 1975/76, in: Lecture Notes in Math., vol. 581, Springer-Verlag, 1975, pp. 518-528.

[29] M. Zähle, Integration with respect to fractal functions and stochastic calculus I, Probab. Theory Related Fields 111 (1998) $333-374$. 